\title{
An analysis of the correlations between the turbulent flow and the sound pressure fields of subsonic jets
}

\author{
C. BOGEY AND C. BA ILLY \\ Laboratoire de Mécanique des Fluides et d'Acoustique, UMR CNRS 5509, Ecole Centrale de Lyon, \\ 69134 Ecully, France
}

(Received 13 March 2006 and in revised form 26 January 2007)

Noise generation is investigated in subsonic isothermal round jets at Mach numbers $M=0.6$ and $M=0.9$, with Reynolds numbers $R e_{D}=1700$ and $R e_{D} \geqslant 10^{5}$, using causality methods on data provided by large-eddy simulations. The correlations between broadband sound pressure signals and broadband turbulence signals along the jet axis and the shear layer are calculated. The normalized correlations are found to be significant between the pressure emitted in the downstream direction and centreline flow quantities. They are much smaller in the cases involving flow quantities along the shear layer, and fall for large emission angles. The maximum correlations obtained between centreline turbulence and downstream sound pressure are observed just at the end of the potential core for time delays corresponding to the times of propagation evaluated along ray paths. They also appear to be lower as the Mach number is reduced, and to be enhanced as the Reynolds number is decreased. These correlation levels can reasonably be attributed to the noise source which is predominant at small emission angles. This source is therefore located on the jet centreline at the end of the potential core, in a flow region which is shown to be characterized by a dominant Strouhal number over a large axial distance, by a strong level of intermittency, and by a high convection velocity. This supports the contention that the downstream jet-noise component is connected to the periodic and intermittent intrusion of vortical structures into the jet core.

\section{Introduction}

More than fifty years after Lighthill's pioneering work in 1952, noise generation mechanisms in subsonic jets are still not well-established (see the review by Tam 1998). This is to a great extent because jet noise theories are closely dependent on the understanding of turbulence, which has significantly evolved. In the 1950s, turbulence was regarded as random, whereas it was considered to be strongly dominated by coherent structures in the 1970s and 1980s. There are also difficulties in identifying sound sources directly from the radiated sound field. Knowledge of the radiated acoustic field, however, sheds light on the noise-generation region. The jet-noisesource strength distribution can be obtained using directional-microphone techniques based on ray-tracing. Chu \& Kaplan (1976) showed that the dominant sound source region in high-subsonic jets is located just downstream of the end of the potential core. The variations of noise properties with jet velocity and emission angle can also bring support to the different jet noise theories. Lush (1971), for example, compared his measurements for subsonic jets with the predictions of Lighthill's theory, in order 
to discuss the validity of the convective amplification factor resulting from the theory (see also the review by Goldstein 1984). The trends exhibited by experimental data at different emission angles, especially in Mollo-Christensen, Kolpin \& Martucelli (1964), Tam, Golebiowski \& Seiner (1996) and Viswanathan (2002), moreover suggest strongly that jet noise at sufficiently high Mach numbers is made of two basic components: one dominating in the downstream direction; another dominating in the sideline direction. The variations of jet noise with Reynolds number are also particularly relevant for characterizing the sound sources. Experiments on jets at low Reynolds numbers have therefore been conducted, for instance by Stromberg, McLaughlin \& Troutt (1980) and Long \& Arndt (1984). Crighton (1981) noticed that the noise radiated by excited jets changes below the Reynolds number $R e_{D}=u_{j} D / v \simeq 10^{5}$ $\left(u_{j}\right.$ is the jet velocity, $c_{0}$ is the speed of sound in the ambient medium, and $v$ is the kinematic molecular viscosity). Simulation results obtained by Bogey \& Bailly (2006b) further showed that, as the Reynolds number decreases, the downstream noise component is not appreciably modified, whereas the sideline noise tends to disappear. Finally, to study jet noise sources from the sound field alone, an accurate description of the near pressure field (e.g. Zaman 1986; Ukeiley \& Ponton 2004) may be especially appropriate. Zaman (1986) discussed sound source locations in terms of the turbulence maxima for a jet at Mach number $M=u_{j} / c_{0}=0.5$.

To investigate jet-noise generation mechanisms, another approach is to search for direct connections between the flow field and the radiated sound field. Specific events generating noise can first be tracked from simultaneous visualizations of the two fields. Pairing and tearing of vortical structures in the shear layer have thus been linked to wave packets radiated by the jets, from experiments by Hileman \& Samimy (2001) and Hileman et al. (2005) and from simulations by Bogey, Bailly \& Juvé (2003). In addition, the quasi-periodic intrusion of vortical structures into the jet at the end of the potential core has been connected to the noise component radiated in the downstream direction (Bogey et al. 2003). Jet-noise sources can also be identified directly by using the causality method in which cross-correlations between flow quantities inside the jet and the radiated sound pressure are calculated. Siddon \& Rackl (1971) and Hurdle, Meecham \& Hodder (1974) selected the incompressible pressure fluctuations as the flow quantity. Hurdle et al. (1974) made measurements on a jet engine, with a pressure probe located one diameter off the jet axis at the end of the potential core. For a jet at $M=0.85$, they observed normalized correlations of 0.139 with the acoustic pressure at an angle of $20^{\circ}$ to the jet axis, but of only 0.006 at $90^{\circ}$. The correlation levels were also found to decrease dramatically as the Mach number decreases, as illustrated by the correlation of 0.025 obtained at $20^{\circ}$ to the jet axis for a jet at $M=0.52$. Other authors determined the cross-correlations between the velocity fluctuations inside the jet and the radiated pressure. The correlations thus measured by Lee \& Ribner (1972), Seiner (1974), Dahan et al. (1978), Juvé, Sunyach \& Comte-Bellot (1980), and Richardz (1980) for jets with Mach numbers in the range $0.3 \leqslant M \leqslant 0.4$ are, however, quite weak. For pressure at $40^{\circ}$ to the jet axis, Lee \& Ribner (1972) obtained a maximum broadband correlation of only 0.02 for an $M=0.3$ jet, whereas Seiner (1974) noted that the correlations at $90^{\circ}$ to the jet axis for an $M=0.33$ jet are too small to be accurately evaluated. In order to enhance the amplitude of the correlations, Lee \& Ribner (1972) and Seiner (1974) filtered their flow and acoustic components, whereas Dahan et al. (1978) and Juvé et al. (1980) used conditional sampling. Seiner (1974) encountered a maximum filtered correlation of 0.12 at $30^{\circ}$ to the jet axis. The correlations obtained from pressure radiated at small angles with respect to the jet direction have allowed us to show 
the source-strength distribution along the jet axis, and to discuss the contributions of the so-called shear-noise and self-noise. The region generating noise at the dominant frequency is found after the end of the potential core. The correlations reported by Schaffar (1979) for a jet at $M=0.98$ exhibited higher levels than those at $M \simeq 0.3$ mentioned above. Schaffar (1979) observed broadband correlations of about 0.06 between acoustic pressure at $30^{\circ}$ to the jet axis and centreline axial velocity just downstream of the jet core. Panda \& Seasholtz (2002) and Panda, Seasholtz \& Elam (2005) measured cross-correlations between the radiated pressure and flow quantities such as density and velocity fluctuations, for jets at Mach numbers 0.8, 0.95, 1.4 and 1.8. They observed higher correlation levels at $M=1.8$, and showed the sharp fall of the maximum correlation with the radiation angle. Furthermore, the correlations between flow quantities along the shear layer and pressure at $30^{\circ}$ to the jet axis were significant only at $M=1.8$.

In the present work, noise generation in subsonic jets is investigated using causality methods for isothermal jets at Mach numbers $M=0.6$ and $M=0.9$, with Reynolds numbers $R e_{D}=1700$ and $R e_{D} \geqslant 10^{5}$, computed by large eddy simulation (LES). Cross-correlations are calculated between the broadband turbulence signals and the broadband sound pressure signals provided directly by the LES. This work is the continuation of earlier studies where different subsonic jets were simulated. The simulations were performed using a solver developed for direct noise computations, with the low-dissipation and low-dispersion numerical schemes designed in Bogey \& Bailly (2004). The LES approach followed is based on an explicit selective filtering for subgrid modelling, as for instance in Rizzetta, Visbal \& Blaisdell (2003), in order to preserve the Reynolds number $R e_{D}$ given by the jet initial conditions. A jet at $M=0.9$ and $R e_{D}=4 \times 10^{5}$ was first considered in Bogey \& Bailly (2006a). Flow and sound properties in agreement with high-Reynolds-number experimental data were obtained. The influence of the inflow conditions and of the subgrid modelling on results was also shown in Bogey \& Bailly $(2005 a, b)$. The effects of the Mach and Reynolds numbers on the noise radiated by jets at Mach numbers 0.6 and 0.9 , with Reynolds numbers varying from 1700 up to $4 \times 10^{5}$, were then described in Bogey \& Bailly (2006b). Two distinguishable components in subsonic jet noise were displayed: a Reynolds-number-dependent noise, predominant for large radiation angles, which is connected to the randomly-developing turbulence, and a deterministic noise, radiating in the downstream direction, which must be associated with a mechanism that is still to be clearly identified. In the present work, we focus on the jets at $M=0.6$ and $M=0.9$, with Reynolds numbers $R e_{D}=1700$ and $R e_{D} \geqslant 10^{5}$. The aim is to study the influence of the Mach and Reynolds numbers on the correlations between broadband acoustic pressure and flow quantities. The correlations considered are more precisely those between the radiated fluctuating pressure, and the fluctuating velocities, the normal stresses, the turbulent kinetic energy and the vorticity norm along the jet axis and the shear layer. They are specially calculated between the jet flow and the sound pressure obtained at the radiation angles, relative to an origin at the end of the potential core, of $40^{\circ}$ and $90^{\circ}$ with respect to the jet direction. In order to characterize the possible sound sources, the centreline turbulence at the end of the jet core, where high correlations with the generated noise are expected to be found according to previous experimental works, will also be examined in terms of spectral contents, intermittency and convection velocity.

The paper is organized as follows. In $\S 2$, the main features of the numerical procedure are presented, the parameters of the different simulations are given, and the observation points are defined. The correlations between the jet flow quantities and 
the radiated pressure are shown in $\S 3$, where properties of the centreline turbulence at the end of the jet potential core are also reported. Section 4 contains concluding remarks.

\section{Simulation parameters}

\subsection{Numerical procedure}

The numerical algorithm and parameters are those of the simulation of a Mach number $M=0.9$, Reynolds number $R e_{D}=4 \times 10^{5}$ jet, referred to as LESac or LESsf in Bogey \& Bailly $(2005 a, b, 2006 a)$. The simulation time is, however, doubled for an accurate evaluation of the correlations between turbulence and noise.

The Cartesian filtered compressible Navier-Stokes equations are solved using numerical schemes with low-dispersion and low-dissipation properties developed in Bogey \& Bailly (2004). Fourth-order thirteen-point finite differences are used for spatial discretization, and an explicit second-order six-stage Runge-Kutta algorithm is applied for time integration. To ensure numerical stability, grid-to-grid oscillations are removed by an explicit fourth-order filtering of the flow variables, which was optimized in the wavenumber space to damp only the short waves discretized by fewer than four points per wavelength. The filtering enables us also to take into account the effects of the subgrid energy-dissipating scales without affecting significantly the resolved scales, as described in detail in Bogey \& Bailly (2006d). This approach was developed to preserve the Reynolds number given by the inflow conditions, which might not be possible using eddy-viscosity subgrid models such as the dynamical Smagorinsky model, as shown in Bogey \& Bailly $(2005 b, 2006 c)$. Finally, in order to compute the radiated noise directly, non-reflective boundary conditions are implemented, with the addition of a sponge zone in the jet at the outflow.

The computational domain is discretized by a 12.5 million point Cartesian grid with 15 points within the range $0 \leqslant y, z \leqslant r_{0}$, and extends radially up to $y=z=15 r_{0}$ from the jet axis. Owing to stretching of the axial mesh size for $x \geqslant 26 r_{0}$, the turbulent flow is computed accurately up to a distance of $x=25 r_{0}$, and the sound field is resolved for Strouhal numbers $S t=f D / u_{j}<2$ ( $f$ is the frequency) up to $x=30 r_{0}$. The simulation times $T$ are doubled with respect to the earlier studies (Bogey \& Bailly $(2006 a, b)$ in order to make sure of the convergence of the cross-correlations. The corresponding Strouhal number is now $D /\left(T u_{j}\right)=4.9 \times 10^{-4}$. The statistics obtained from the time period $T$ are found not to differ appreciably from those obtained from the time period $(2 / 3) \times T$. Spectra and cross-correlations are performed from velocity and pressure signals of total time $T_{s}$ such as $D /\left(T_{s} u_{j}\right)=5.2 \times 10^{-4}$ in terms of minimal Strouhal number. In addition, the pressure signals are filtered using moving averaging in order to attenuate the low-frequency components with Strouhal numbers $S t<0.05$. For the computation of the sound spectra, they are divided into 449 overlapping sections of time $T_{s} / 225$, windowed by a Hanning function.

\subsection{Present simulations}

Four isothermal round jets are considered. Their initial conditions are identical except for the diameter and the jet exit velocity, yielding Mach numbers of 0.6 and 0.9 and Reynolds numbers $R e_{D}=1700$ and $R e_{D} \geqslant 10^{5}$ (table 1 ). The LESm09hre simulation is the jet simulation at Mach $M=0.9$ and at the high Reynolds number $\operatorname{Re}_{D}=4 \times 10^{5}$, also referred to as LESac or as LESsf in Bogey \& Bailly $(2005 a, b, 2006 a)$. In the LESm09re1700 simulation, the Mach number of 0.9 is maintained, but the Reynolds number is decreased to $R e_{D}=1700$. In the LESm06hre and LESm06re1700 


\begin{tabular}{lccrcc} 
& $M$ & \multicolumn{1}{c}{$\boldsymbol{R e}_{D}$} & $x_{c}$ & $M_{40}(x, y)$ & $M_{90}(x, y)$ \\
LESm09hre & 0.9 & $4 \times 10^{5}$ & $10.2 r_{0}$ & $\left(28 r_{0}, 15 r_{0}\right)$ & $\left(10 r_{0}, 15 r_{0}\right)$ \\
LESm09re1700 & 0.9 & $1.7 \times 10^{3}$ & $15.8 r_{0}$ & $\left(29 r_{0}, 11 r_{0}\right)$ & $\left(16 r_{0}, 15 r_{0}\right)$ \\
LESm06hre & 0.6 & $2.7 \times 10^{5}$ & $9.5 r_{0}$ & $\left(27 r_{0}, 15 r_{0}\right)$ & $\left(10 r_{0}, 15 r_{0}\right)$ \\
LESm06re1700 & 0.6 & $1.7 \times 10^{3}$ & $13.3 r_{0}$ & $\left(29 r_{0}, 13 r_{0}\right)$ & $\left(13 r_{0}, 15 r_{0}\right)$
\end{tabular}

TABLE 1. Mach and Reynolds numbers of the jets, location of the end of the potential core $x_{c}$ and of the observation points $M_{40}$ and $M_{90}$ in the different simulations.

simulations, the Mach number is reduced to $M=0.6$. The former simulation is at a high Reynolds number $R e_{D} \geqslant 10^{5}$, whereas the latter is at the low Reynolds number $R e_{D}=1700$ as in LESm09re1700.

In all the simulations, the inflow parameters (shear-layer thickness, forcing) are identical. Mean profiles of velocities, pressure and density are imposed at the jet inflow boundary. The axial velocity is given by a hyperbolic-tangent profile describing an annular shear layer of radius $r_{0}$ and of momentum thickness $\delta_{\theta}$, with a ratio $\delta_{\theta} / r_{0}=0.05$. Radial and azimuthal velocities are set to zero, pressure is set to the ambient pressure, and the mean density profile is obtained using a CroccoBusemann relation for an isothermal jet. To trip the turbulent transition, small random disturbances are added to the velocity profiles in the shear-layer zone. Note that the influence of the inflow conditions and forcing on the jet flow and sound fields was studied in Bogey \& Bailly (2005a).

Because of the LES limitations, the turbulent scales smaller than the filtering cutoff length, and consequently their associated noise, are lacking. In Bogey \& Bailly $(2006 d)$, the filtering cutoff wavenumber was estimated as $k=12 / r_{0}$ in the present jets, which provides a cutoff Strouhal number of $S t=12 / \pi=3.8$. The sound field being resolved up to $S t=2$, the turbulent scales involved in the generation of the computed pressure are therefore expected to be calculated. Furthermore, given the predominant Strouhal-number range of subsonic jet noise, the effects of the LES cutoff frequency should be small on the broadband correlations between turbulence and noise.

\subsection{Study specifications}

Snapshots of the pressure fields generated by the jets were presented in Bogey \& Bailly (2006b). The properties of the sound radiated in the downstream and sideline directions were also described in detail in order to characterize the two jet-noise components. The first component appears as a low-Strouhal-number peak in the spectra obtained in the jet direction. In the present jets, it was found to dominate at about $30^{\circ}$ to the jet axis, with Strouhal number peaks of $S t \simeq 0.3$ at high Reynolds numbers $R e_{D} \geqslant 10^{5}$, and of $S t \simeq 0.2$ at $R e_{D}=1700$. The second component was observed to be predominant for large radiation angles. It is broadband and strongly dependent on the Reynolds number.

\subsubsection{Definition of the observation points}

To study noise generation in the present jets using cross-correlations between turbulence and noise, pressure and turbulence quantities are recorded at the observations points represented in figure 1 . The pressure signals are obtained along the lines defined by $y=15 r_{0}$ and $z=0$, and by $x=29 r_{0}$ and $z=0$. The turbulence signals are provided along the jet axis at $y=z=0$ and along the shear layer at $y=r_{0}$ and $z=0$. 


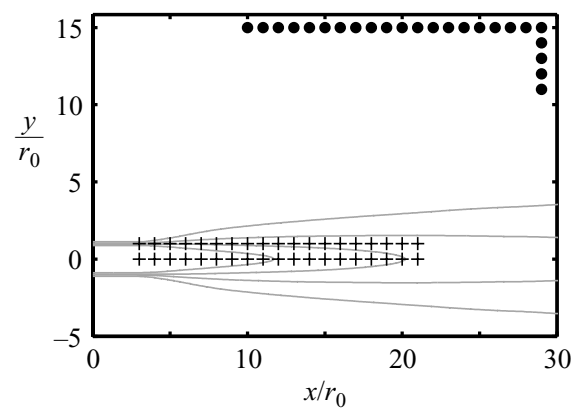

FIGURE 1. Visualization of the observation points: + , in the jet; $\bullet$, in the acoustic field. The grey lines represent the contours of mean axial velocities $[0.1,0.36,0.63,0.9] \times u_{j}$, in the plane $z=0$, for the LESm09hre jet.

The turbulence quantities considered in the jet flow are the velocity components $u^{\prime}, v^{\prime}$ and $w^{\prime}$, the normal stresses $u^{\prime 2}, v^{\prime 2}$ and $w^{\prime 2}$, the turbulent kinetic energy $k=\left(u^{\prime 2}+v^{\prime 2}+w^{\prime 2}\right) / 2$ and the vorticity norm $|\omega|$. These quantities are correlated with the radiated fluctuating pressure $p^{\prime}$ to determine the cross-correlation functions Cup, Cvp, Cwp, Cuup, Cvvp, Cwwp, $C k p$ and $C|\omega| p$. The normalized correlations between the flow quantities $u^{\prime}, u^{\prime 2}, k$ and $|\boldsymbol{\omega}|$ at point $\boldsymbol{x}_{1}$ and fluctuating pressure at point $\boldsymbol{x}_{2}$ are calculated in the following way:

$$
\begin{aligned}
\operatorname{Cup}\left(\boldsymbol{x}_{1}, \boldsymbol{x}_{2}, \tau\right) & =\frac{\left\langle u^{\prime}\left(\boldsymbol{x}_{1}, t\right) p^{\prime}\left(\boldsymbol{x}_{2}, t+\tau\right)\right\rangle}{\left\langle u^{\prime 2}\left(\boldsymbol{x}_{1}, t\right)\right\rangle^{1 / 2}\left\langle p^{\prime 2}\left(\boldsymbol{x}_{2}, t\right)\right\rangle^{1 / 2}}, \\
\operatorname{Cuup}\left(\boldsymbol{x}_{1}, \boldsymbol{x}_{2}, \tau\right) & =\frac{\left\langle u^{\prime 2}\left(\boldsymbol{x}_{1}, t\right) p^{\prime}\left(\boldsymbol{x}_{2}, t+\tau\right)\right\rangle}{\left\langle u^{\prime 4}\left(\boldsymbol{x}_{1}, t\right)\right\rangle^{1 / 2}\left\langle p^{\prime 2}\left(\boldsymbol{x}_{2}, t\right)\right\rangle^{1 / 2}}, \\
\operatorname{Ckp}\left(\boldsymbol{x}_{1}, \boldsymbol{x}_{2}, \tau\right) & =\frac{\left\langle k\left(\boldsymbol{x}_{1}, t\right) p^{\prime}\left(\boldsymbol{x}_{2}, t+\tau\right)\right\rangle}{\left\langle k^{2}\left(\boldsymbol{x}_{1}, t\right)\right\rangle^{1 / 2}\left\langle p^{\prime 2}\left(\boldsymbol{x}_{2}, t\right)\right\rangle^{1 / 2}}, \\
C|\boldsymbol{\omega}| p\left(\boldsymbol{x}_{1}, \boldsymbol{x}_{2}, \tau\right) & =\frac{\left\langle|\boldsymbol{\omega}|\left(\boldsymbol{x}_{1}, t\right) p^{\prime}\left(\boldsymbol{x}_{2}, t+\tau\right)\right\rangle}{\left\langle|\boldsymbol{\omega}|^{2}\left(\boldsymbol{x}_{1}, t\right)\right\rangle^{1 / 2}\left\langle p^{\prime 2}\left(\boldsymbol{x}_{2}, t\right)\right\rangle^{1 / 2}},
\end{aligned}
$$

where $\langle\cdot\rangle$ denotes time averaging, and $\tau$ is the time delay between the turbulence and the pressure signals.

The aim is to investigate the correlations between the full turbulent field and the broadband sound pressure signals of the four jets, in order to track connections at different Mach and Reynolds numbers. The correlations are calculated with flow quantities that are usually not available experimentally, such as turbulent kinetic energy or vorticity. The simulation results will be compared with the broadband correlations reported by Schaffar (1979) for a jet at $M=0.98$ and by Panda \& Seasholtz (2002) and Panda et al. (2005) for jets at Mach numbers 0.95, 1.4 and 1.8. Lee \& Ribner (1972), Seiner (1974), Dahan et al. (1978), Juvé et al. (1980) and Richardz (1980) also measured flow-noise correlations, but they dealt with jets at Mach numbers $0.3 \leqslant M \leqslant 0.4$, and obtained very low correlation levels. It would be interesting to compute correlations from the LES data using filtering of the flow and acoustic components or conditional sampling, in order to enhance the correlation levels or to focus on a particular noise mechanism. Another idea is to correlate the sound pressure only with the part of the turbulence which is contributing to the radiated noise. The acoustically relevant part of the flow field may be very different from the latter, as shown by Freund (2001) for instance. 

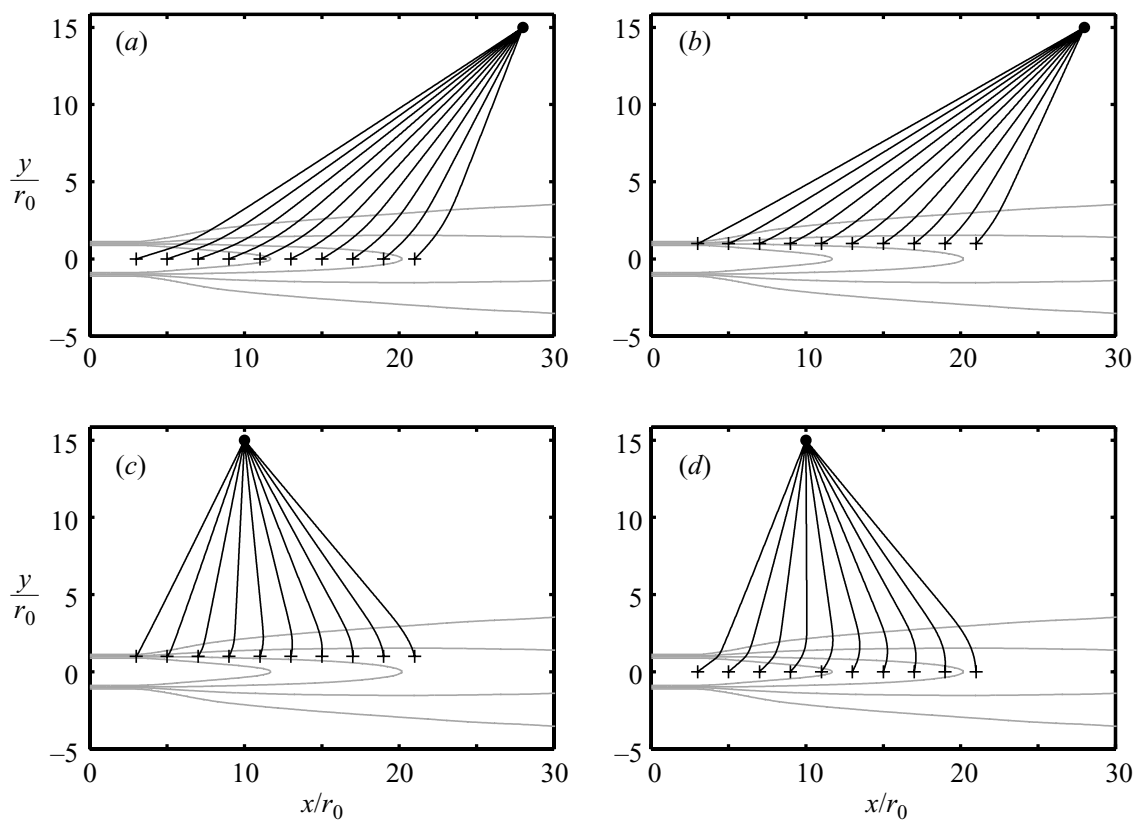

FiguRE 2. Sketch of the four cases: (a) $M_{40}$ /axis, (b) $M_{40} /$ shear-layer, (c) $M_{90} /$ shear-layer, (d) $M_{90} /$ axis; in which correlations are calculated between pressure at points $M_{40}$ or $M_{90}(\bullet)$, and flow quantities along the jet axis at $y=0$ or along the shear layer at $y=r_{0}(+)$. The black lines represent calculated ray paths, and the grey lines show the contours of mean axial velocities $[0.1,0.36,0.63,0.9] \times u_{j}$, in the plane $z=0$, for the LESm09hre jet.

In the present jets, the potential core length is not constant owing to Mach- and Reynolds-number effects. The location of the end of the potential core $x_{c}$, determined from the centreline mean axial velocity $u_{c}$ using $u_{c}\left(x_{c}\right)=0.95 u_{j}$, varies from $x_{c}=9.5 r_{0}$ up to $x_{c}=15.8 r_{0}$ (table 1 ). To obtain results at fixed radiation angles, two points $M_{40}$ and $M_{90}$ defining angles of $40^{\circ}$ and $90^{\circ}$ from the jet axis, relative to an origin at the end of the jet core, are selected from among the observation points in the acoustic field. Their locations are given in table 1 for the different simulations.

In the next section, four cases will be investigated, depending on whether correlations are calculated between pressure at points $M_{40}$ or $M_{90}$, and turbulence along the jet axis or along the shear layer. They are represented in figure 2, and are referred to as $M_{40} /$ axis, $M_{40} /$ shear-layer, $M_{90} /$ shear-layer and $M_{90} /$ axis. Another case will also be considered to study the angular dependence of the results, by correlating the centreline turbulence at $x=x_{c}$ with the pressure at the different observation points in the acoustic field presented in figure 1.

Points $M_{40}$ and $M_{90}$ are located in the near acoustic field, and the acoustic pressure at these points might be contaminated by the aerodynamic pressure. Arndt, Long \& Glauser (1997) showed that the pressure measured at the outer edge of a jet, typically at $r=D$, is basically aerodynamic. The magnitude of the aerodynamic pressure, however, decreases rapidly with the distance from the jet axis, and Arndt et al. (1997) and Coiffet et al. (2006) noticed that the near-field pressure fluctuations are acoustic for wavenumbers $k r>2.0$. At the radial distance $r=15 r_{0}$, this condition yields $S t>0.047$ at Mach 0.9, and $S t>0.070$ at Mach 0.6, which was confirmed in the measurements of Bogey et al. (2007). In the present study, the dominant part of 

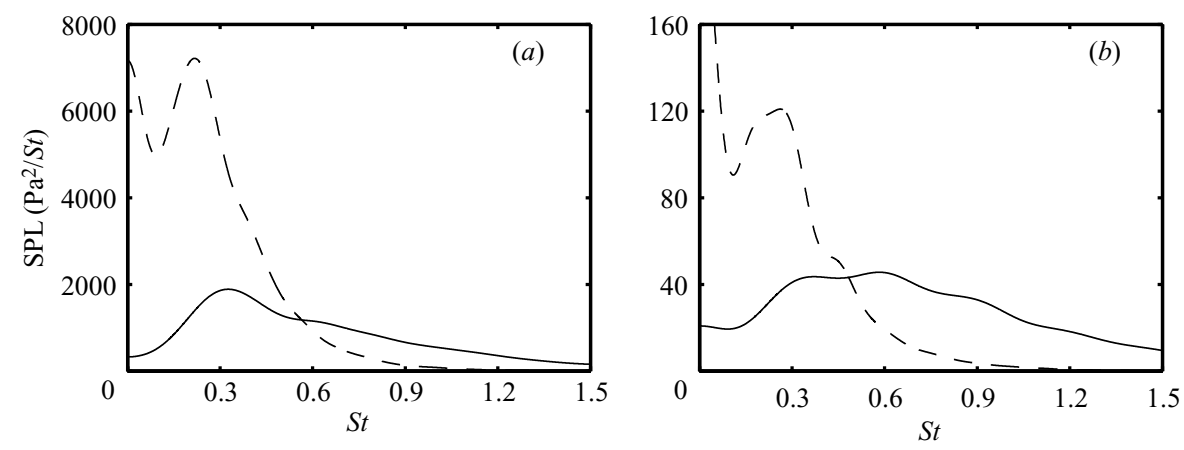

FIGURE 3. Pressure spectra obtained at point $M_{40}$ as a function of Strouhal number $S t=$ $f D / u_{j}$ : (a) Mach 0.9 jets, $(b)$ Mach 0.6 jets; at: - - high Reynolds number; ---, $\operatorname{Re}_{D}=1700$.

the pressure spectra is obtained for $S t>0.1$. It is therefore not appreciably affected by aerodynamic disturbances. Moreover, since the pressure signals are filtered to attenuate the components with $S t<0.05$, possible low-frequency contributions of the aerodynamic pressure are reduced.

\subsubsection{Sound pressure spectra}

The properties of the sound fields at points $M_{40}$ and $M_{90}$, for which pressure will be correlated with the jet turbulence, are checked. Note that the levels of the sound spectra at $M_{40}$ and $M_{90}$ cannot be directly compared, because, given the locations of these points (table 1), the distances from the sound sources to $M_{40}$ and $M_{90}$ are expected to differ significantly.

The spectra obtained at point $M_{40}$ at the radiation angle of $40^{\circ}$ are presented in figure 3(a) for the $M=0.9$ jets and in figure 3(b) for the $M=0.6$ jets. At $M=0.9$, the spectra are clearly dominated by frequency peaks typical of the downstream noise component around $S t \simeq 0.25$. At $M=0.6$, the downstream component peak is also apparent for the jet at low Reynolds number. However, for the jet at high Reynolds number, the contributions of the two jet-noise components cannot be distinguished.

In the spectra obtained at $R e_{D} \geqslant 10^{5}$, no significant aerodynamic components are observed. At $R e_{D}=1700$, low-frequency aerodynamic components are visible for $S t<0.1$. In LESm09re1700 and LESm06re1700, points $M_{40}$ are indeed located closer to the jet axis, at $r=11 r_{0}$ and $r=13 r_{0}$, respectively. This might have slight effects on the correlations involving pressure at $M_{40}$ for these two jets. It must be noted, however, that the observation points in the jet are located at $x \leqslant 21 r_{0}$, whereas points $M_{40}$ are farther downstream at $x=29 r_{0}$.

The pressure spectra obtained at point $M_{90}$ in the sideline direction are presented in figures $4(a)$ and $4(b)$ for the jets at Mach 0.9 and 0.6. The dramatic influence of the Reynolds number on the sideline spectra is observed. As the Reynolds number decreases, a significant part of the high-frequency noise disappears. Consequently, the peak frequency becomes lower, down to Strouhal number $S t \simeq 0.2$ at Reynolds number $R e_{D}=1700$. At a given Reynolds number, the shapes of the spectra obtained for the jets at $M=0.9$ and $M=0.6$ are, however, similar. The similarity of the spectra was especially shown in Bogey \& Bailly (2006b). In that paper, the variations of sideline sound spectra with the velocity were also found to follow a $u_{j}^{7.5}$ scaling, in agreement with experimental data of Zaman \& Yu (1985), at high Reynolds number, but also at $R e_{D}=1700$. In both cases, the characteristics of sideline spectra thus 

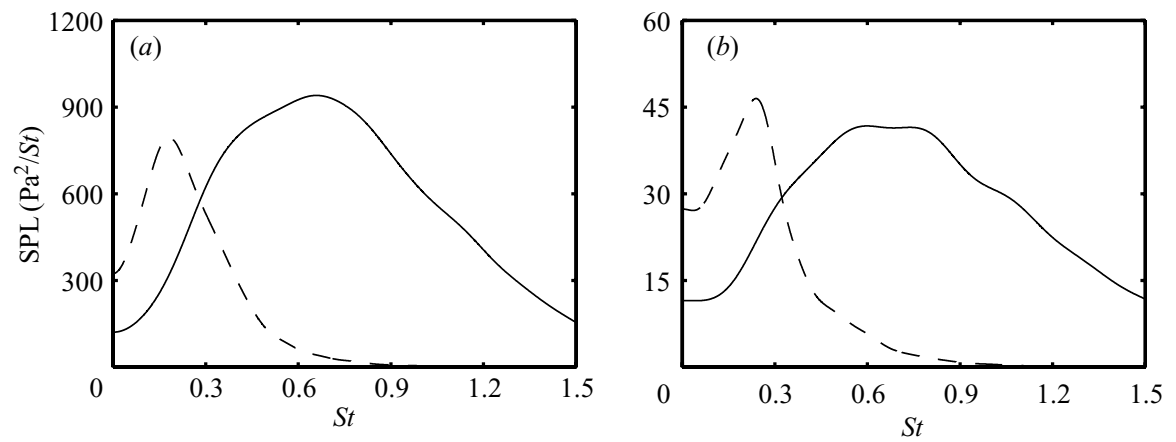

FiguRE 4. Pressure spectra obtained at point $M_{90}$ as a function of Strouhal number $S t=$ $f D / u_{j}:(a)$ Mach 0.9 jets, (b) Mach 0.6 jets; at: $R e_{D}=1700$.

appear to be those of the broadband noise component attributed to the turbulence developing randomly in the jet.

\subsubsection{Time propagation along the ray paths}

To evaluate in the next section the possible connections between flow-noise correlations and sound generation, the propagation times between the source points in the jet flow and the observation points in the acoustic field are finally calculated. The ray-tracing equations, given for instance by Candel (1977), are then solved. They are integrated in time using a fourth-order Runge-Kutta algorithm with $\Delta t=0.1 r_{0} / c_{0}$. To compute the propagation times with a good accuracy, the mean flow fields provided by the LES are used as the background medium. In addition, the derivatives of the mean flow quantities are calculated using standard centred fourth-order finite-differences, and their values along the rays are obtained using Lagrangian polynomials.

The ray paths between the points located along the jet axis and the shear layer, and points $M_{40}$ and $M_{90}$ are determined, in order to deal with the four cases considered. They are represented in figure 2 for the LESm09hre jet. The influence of the mean flow on wave propagation is visible. In particular, the refraction effects on the acoustic waves travelling in the downstream direction in the case $M_{40} /$ axis appear clearly in figure $2(a)$. The propagation times $\tau_{p}$ from the source to the observation points are then evaluated along the ray paths. Those obtained between the centreline points and points $M_{40}$ and $M_{90}$ for the LESm09hre jet are shown in figure 5. To illustrate the effects of mean flow, these propagation times $\tau_{\text {ray }}$ calculated along the ray paths are compared to the times $\tau_{c_{0}}$ obtained for a straight propagation at the sound speed $c_{0}$. Slight but noticeable differences are observed, especially for the downstream point $M_{40}$ in figure 5(a). In this case, the time $\tau_{\text {ray }}$ is lower than $\tau_{c_{0}}$ owing to the convection of sound waves by the jet flow.

\section{Results}

\subsection{Correlations between pressure at $M_{40}$ and jet turbulence}

\subsubsection{Case $M_{40} /$ axis}

The normalized correlations between the fluctuating pressure at point $M_{40}$ at $40^{\circ}$ to the jet axis and the turbulence signals $u^{\prime}, u^{\prime 2}, v^{\prime 2}, w^{\prime 2}, k$ and $|\omega|$ along the jet axis are represented in figures 6 and 7 for the different jets. The correlations with the fluctuating velocities $v^{\prime}$ and $w^{\prime}$ are not shown because they are found to be very low. 

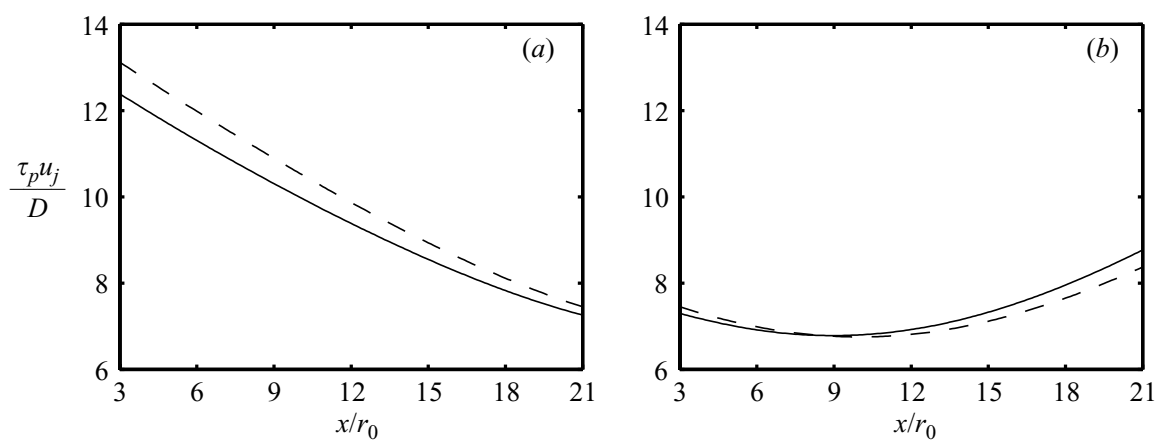

FIGURE 5. Propagation time $\tau_{p}$ between the centreline points at $x / r_{0}$ and $(a)$ point $M_{40}$, (b) point $M_{90}$, for the LESm09hre jet. Time calculated:,$- \tau_{\text {ray }}$ along the ray paths using the LES mean flow; ---, $\tau_{c_{0}}$ along straight lines at the sound speed $c_{0}$.

(i)

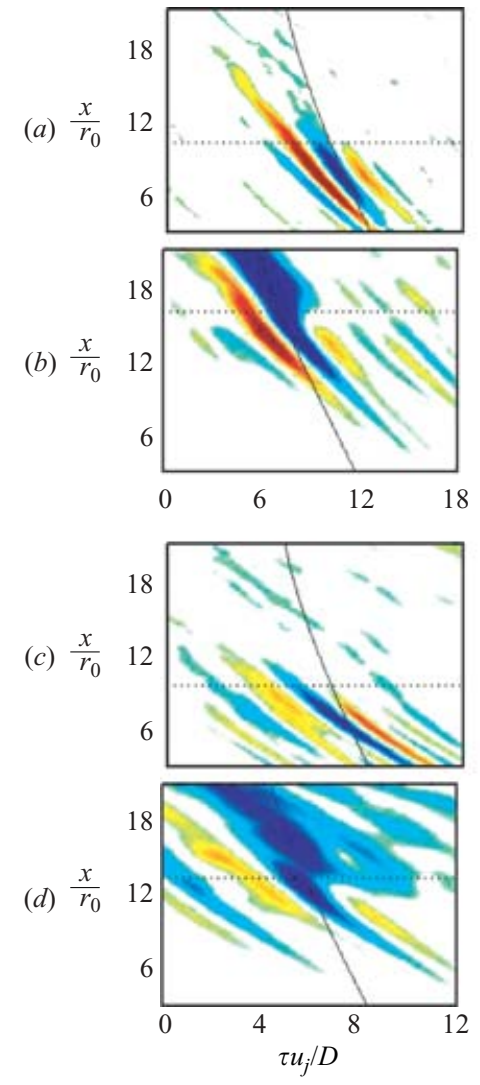

(ii)
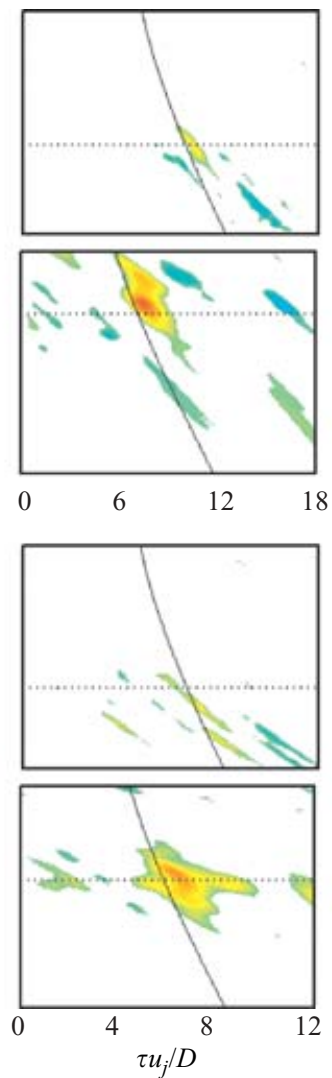

(iii)
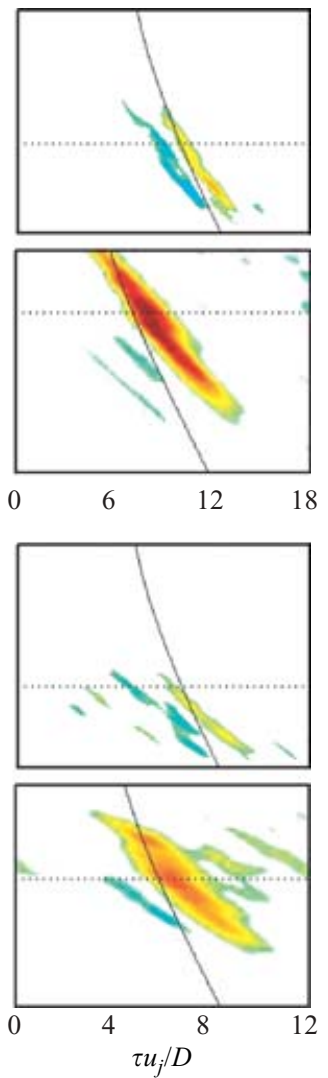

Figure 6. Case $M_{40}$ /axis. Normalized correlations between pressure at $M_{40}$ and flow quantities along the jet axis: (i) Cup, (ii) Cuup, (iii) Cvvp, for (a) LESm09hre, (b) LESm09re1700, (c) LESm06hre, (d) LESm06re1700 ( $X$-axis: time delay $\tau u_{j} / D, Y$-axis: axial location $x / r_{0}$ along the jet axis). The colour scale is defined from -0.14 to 0.14 , with white in the range $[-0.035,0.035]$. The solid lines represent the propagation time $\tau_{\text {ray }}$ along the ray paths between centreline points and point $M_{40}$. The dotted lines show the end of the potential core. 
(i)
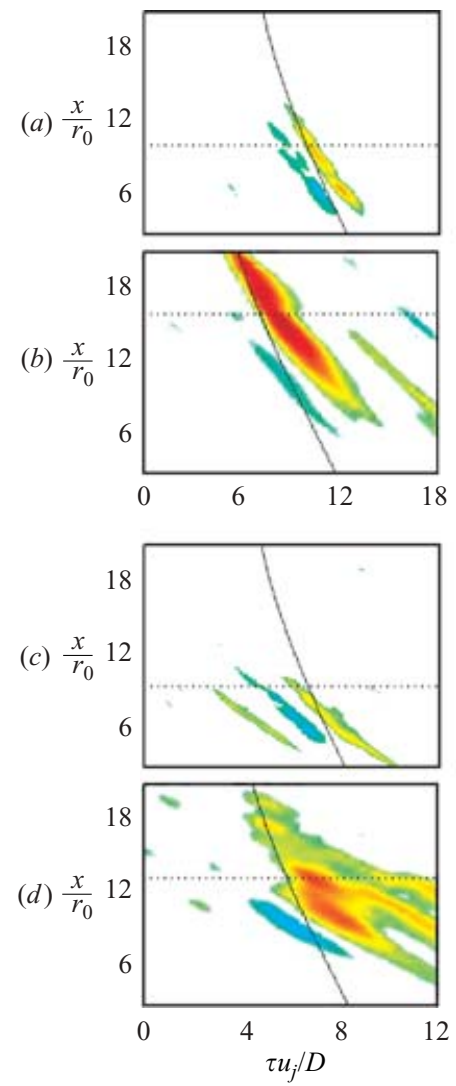

(ii)
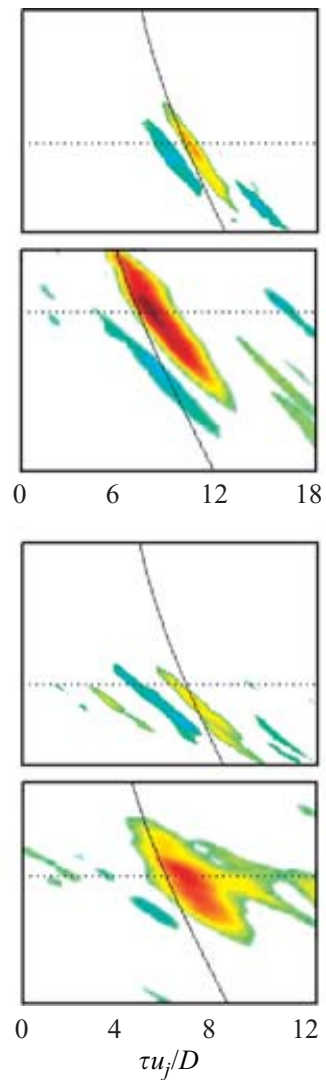

(iii)
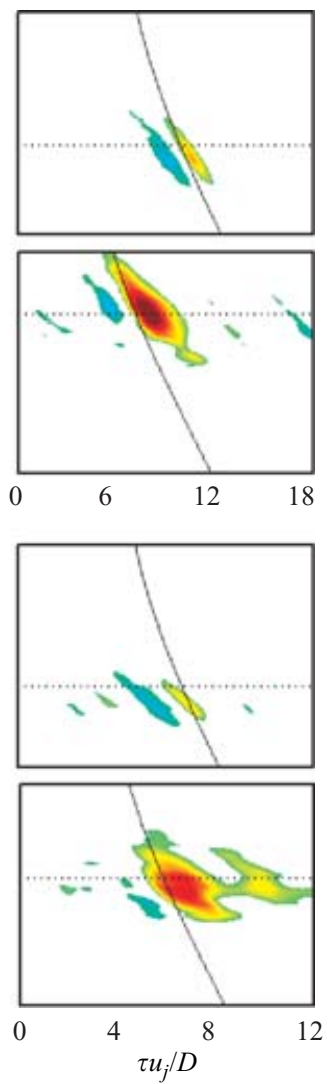

FIGURE 7. Case $M_{40}$ /axis. Normalized correlations between pressure at $M_{40}$ and flow quantities along the jet axis: (i) $C w w p$, (ii) $C k p$, (iii) $C|\omega| p$, for (a) LESm09hre, (b) LESm09re1700, (c) LESm06hre, $(d)$ LESm06re1700. See caption of figure 6 for colour scale and details.

At a given Reynolds number, the correlation maps provided by the jets at Mach 0.9 and 0.6 are very similar. This is visible both at $R e_{D} \geqslant 10^{5}$ (figures $6(a, c)$ and $7(a, c)$ ), and at $R e_{D}=1700$ (figures $6(b, d)$ and $7(b, d)$ ). The correlations presented display large zones with levels noticeably higher than 0.04 . The correlations $C u p$ with the axial fluctuating velocity $u^{\prime}$ show in figure 6(i) significant values of both positive and of negative signs, whereas the correlations with the Reynolds stresses, the turbulent kinetic energy and the vorticity norm are dominated by positive values, especially at the low Reynolds number $R e_{D}=1700$.

The higher levels of correlation are observed in the vicinity of the end of the potential core, which is indicated by a horizontal dotted line. They also occur for time delays close to the propagation times $\tau_{\text {ray }}$ along the ray paths between the centreline points and point $M_{40}$, which are given by a solid line. The maximum correlations are even located nearly at the point of intersection between the solid and dotted lines. For the correlations with the axial velocity $u^{\prime}$, the peak at this point is negative, as illustrated in figure 6(b)(i) for the LESm09re1700 jet. The significant correlations Cup of positive sign are thus mainly found for time delays $\tau<\tau_{\text {ray }}$. For the other correlations, involving the Reynolds stresses, the turbulence kinetic energy and the vorticity norm, the peak is positive and is clearly located at $x \simeq x_{c}$ and $\tau \simeq \tau_{\text {ray }}$, as 
(i)

(a)

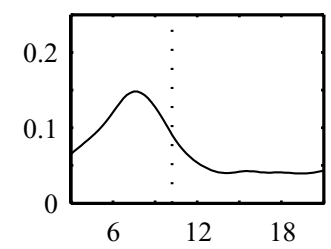

(b)

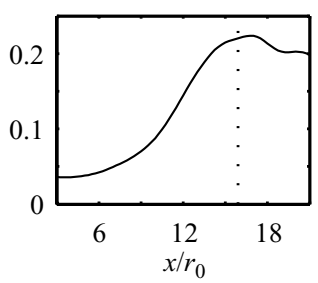

(ii)
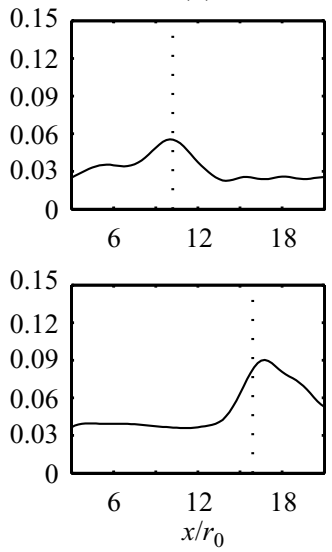

(iii)
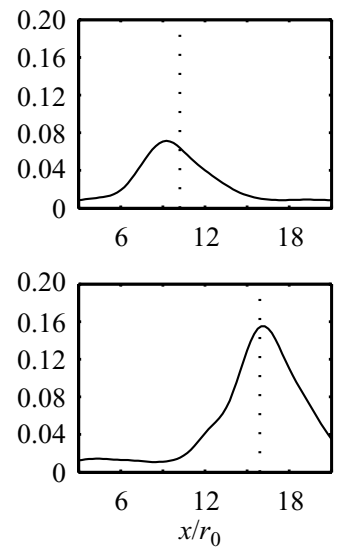

Figure 8. Case $M_{40} /$ axis. Axial variation of peak correlation between pressure at $M_{40}$ and flow quantities at $x / r_{0}$ along the jet axis. Peaks of (i) $-C u p$, (ii) $C u u p$, (iii) $C|\omega| p$, for (a) LESm09hre, (b) LESm09re1700. The dotted lines show the end of the potential core.

(i)

(a)

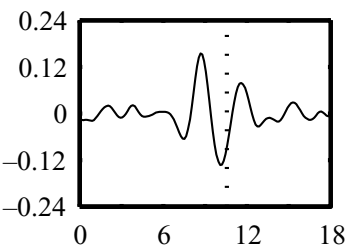

(b)

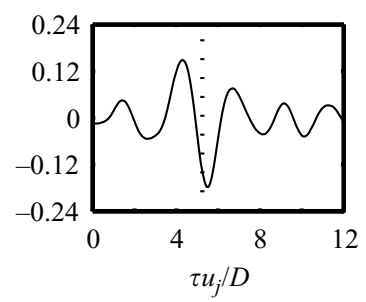

(ii)
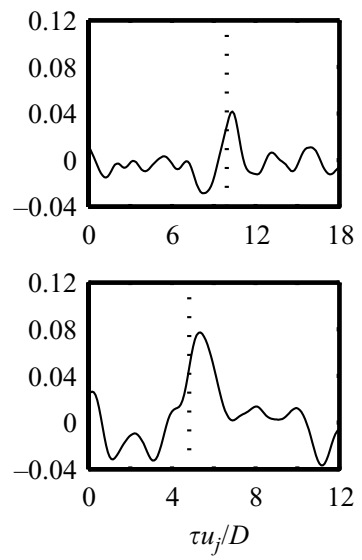

(iii)
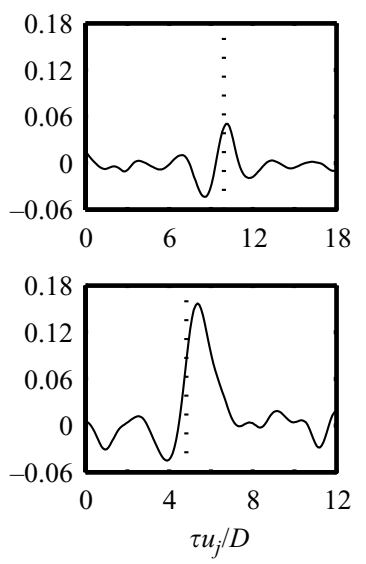

FIgURE 9. Case $M_{40}$ /axis. Normalized correlations between pressure at $M_{40}$ and flow quantities on the jet axis: (i) Cup for a centreline point at $x=x_{c}-2 r_{0}$, (ii) $C u u p$ and (iii) $C|\boldsymbol{\omega}| p$ for a point at $x=x_{c}$, for $(a)$ LESm09hre, $(b)$ LESm09re1700 ( $X$-axis: time delay $\left.\tau u_{j} / D\right)$. The dotted lines represent the propagation time $\tau_{\text {ray }}$ between the centreline point and point $M_{40}$.

for instance in figure 7(b)(iii). Moreover, the correlation fields seem to be only very approximately aligned with the propagation times $\tau_{\text {ray }}$. This point will be discussed in $\S 3.3 .3$ dealing with the convection velocity along the jet axis. It implies nevertheless that low levels of cross-correlation are found after the potential core for $\tau>\tau_{\text {ray }}$.

The space/time position of the maximum correlations is investigated quantitatively in figures 8 and 9. In figure 8, the axial profiles of the peaks of (-Cup), Сuиp and $C|\boldsymbol{\omega}| p$ are represented for the two jets at Mach 0.9, similar results being obtained for the jets at Mach 0.6. In all the cases, the maximum is observed near the end of the potential core. The axial location of the maximum correlations is close to $x=x_{c}$ for the jet at $R e_{D}=1700$ in figure $8(b)$. For the jet at Reynolds number $R e_{D} \geqslant 10^{5}$, the maximum correlations appear slightly upstream, e.g. in figure $8(a)$ (i) for the peak of $(-C u p)$. 


$\begin{array}{lcccccc} & (- \text { Cup })_{m} & (\text { Cuup })_{m} & (\text { Cvvp })_{m} & (\text { Cwwp })_{m} & (\text { Ckp })_{m} & (C|\omega| p)_{m} \\ \text { LESm09hre } & 0.155 & 0.059 & 0.084 & 0.076 & 0.082 & 0.077 \\ \text { LESm09re1700 } & 0.230 & 0.098 & 0.144 & 0.131 & 0.145 & 0.168 \\ \text { LESm06hre } & 0.153 & 0.057 & 0.059 & 0.064 & 0.071 & 0.063 \\ \text { LESm06re1700 } & 0.155 & 0.087 & 0.103 & 0.102 & 0.115 & 0.118\end{array}$

TABLE 2. Case $M_{40}$ /axis. Maximum correlations between pressure at point $M_{40}$ and flow quantities along the jet axis. The subscript $m$ indicates maximum.

The cross-correlation functions between fluctuating pressure at point $M_{40}$ and velocity $u^{\prime}$ at $x=x_{c}-2 r_{0}$, stress $u^{\prime 2}$ and vorticity norm $|\omega|$ at $x=x_{c}$ on the jet centreline are given in figure 9 for the two jets at Mach 0.9. The shapes of the correlation functions Сuиp and $C|\boldsymbol{\omega}| p$ are similar, and exhibit a peak at a time delay corresponding nearly to the propagation time $\tau_{\text {ray }}$. Those of the correlation functions Cup are different with a positive peak followed by a negative peak located at approximately $\tau=\tau_{\text {ray }}$, as shown in figure $9(b)$ (i) for the LESm09re1700 jet. The present correlation curves are in good agreement with the experimental data obtained by Schaffar (1979) for a jet at $M=0.98$ between pressure at $30^{\circ}$ to the jet axis and centreline turbulence at the end of the jet core.

As for the levels of the maximum correlations between fluctuating pressure at point $M_{40}$ and centreline turbulence, they are given in table 2 with the maxima of (-Cup), Cuup, Cvvp, Cwwp, Ckp and $C|\omega| p$ for the four simulated jets. Their variations with the Mach and Reynolds numbers are thus shown. In all the cases reported, the correlations decrease as the Mach number is reduced from $M=0.9$ to $M=0.6$. This trend is in agreement with the experimental data of Hurdle et al. (1974) and Panda et al. (2005); however, the variations of the present correlations with the Mach number appear smaller than in experiments. For the jets at high Reynolds number, the maxima of Сиир are indeed 0.059 at Mach 0.9 and 0.057 at Mach 0.6, while at $R e_{D}=1700$ the maxima are, respectively, 0.098 and 0.087 . Note nevertheless that Hurdle et al. (1974) correlated the far-field pressure with the incompressible pressure fluctuations, and that Panda \& Seasholtz (2002) and Panda et al. (2005) dealt with high-velocity jets at Mach numbers 0.95, 1.4 and 1.8. In the present work, the correlations are also higher at Reynolds number $R e_{D}=1700$ than at $R e_{D} \geqslant 10^{5}$. This can reasonably be attributed to the disappearance of a part of the small turbulent scales, and of a part of the high-frequency noise components, as the Reynolds number decreases. The effects of the Mach and Reynolds numbers are shown in figure 10 by the axial variations of the peak of $C|\omega| p$ for the different jets.

In table 2, the higher values of correlation are obtained for the maxima of ( - Cup) which are around 0.15 . For a given jet, the maximum correlations calculated from the signals of $u^{\prime 2}, v^{\prime 2}, w^{\prime 2}, k$ and $|\omega|$ are moreover rather close, with, for instance, levels between 0.06 and 0.08 for the LESm09hre jet. These turbulence signals can therefore be expected to display a common characteristic. It is difficult to check the present correlation levels from the experimental data in the literature. Lee \& Ribner (1972), Seiner (1974), Dahan et al. (1978), Juvé et al. (1980) and Richardz (1980) provided very low broadband cross-correlations for jets at low Mach numbers $M \leqslant 0.4$. The most relevant experimental data seem to be the broadband correlations provided by Schaffar (1979) for a jet at $M=0.98$, between fluctuating pressure at $30^{\circ}$ to the jet axis and signals of $u^{\prime}$ and $u^{\prime 2}$ on the jet axis. Schaffar (1979) reported in this case peaks of (-Cup) and Сuиp of about 0.06 and 0.04, respectively. Panda et al. (2005) also 


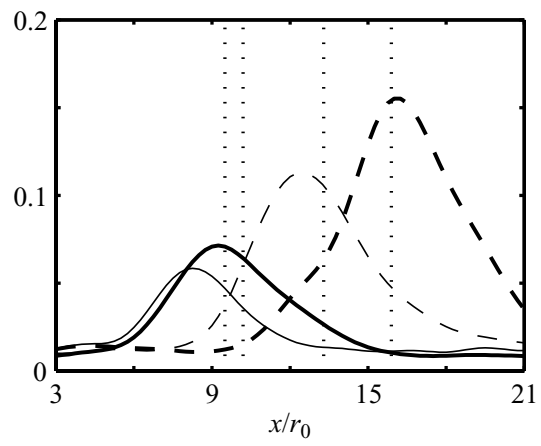

FIgURE 10. Case $M_{40}$ /axis. Axial variation of peak correlation between pressure at $M_{40}$ and vorticity norm at $x / r_{0}$ along the jet axis, for - , LESm09hre; - - , LESm09re1700; - LESm06hre; ---, LESm06re1700. The dotted lines show the end of the potential core for the different jets.

$\begin{array}{lllllll}u_{\text {noise }}^{\prime} / u_{j} & 0 & 0.01 & 0.02 & 0.03 & 0.04 & 0.05 \\ (- \text { Cup })_{m} & 0.155 & 0.154 & 0.148 & 0.141 & 0.132 & 0.123 \\ (\text { С иup })_{m} & 0.059 & 0.059 & 0.057 & 0.055 & 0.052 & 0.049\end{array}$

TABLE 3. Case $M_{40}$ /axis. Maximum correlations when a random velocity of intensity $u_{\text {noise }}^{\prime} / u_{j}$ is introduced in the jet centreline for LESm09hre.

obtained a $C$ up peak of 0.064 in a jet at $M=0.95$ for a radiation angle of $30^{\circ}$. The experimental values are significantly lower than our results for the LESm09hre jet.

There may be several reasons for this discrepancy. We can mention the features of the numerical simulations which can be expected to increase the correlation levels with respect to the experiments. The LES signals are first limited in terms of frequency, with cutoff Strouhal numbers of $S t=3.8$ for the turbulence, and $S t=2$ for the sound field, as noted previously. Secondly, in the simulations, the jet inflow is laminar, and the velocity disturbances on the jet axis are negligible for $x \leqslant 4 r_{0}$ (Bogey \& Bailly 2006d), whereas the experimental jets are at high Reynolds numbers, with for instance $R e_{D}=9 \times 10^{5}$ (Schaffar 1979), and must be initially fully turbulent according to Zaman (1985). Parasitic velocity fluctuations are thus probably in the core of these jets. For example in Lau, Morris \& Fisher (1979) and in Arakeri et al. (2003), around $2 \%$ of turbulence were measured on the jet axis just after the nozzle. To illustrate the influence of these disturbances on the correlations, a random velocity of intensity $0 \leqslant u_{\text {noise }}^{\prime} \leqslant 0.05 u_{j}$ is added to the centreline velocity $u^{\prime}$ calculated for the LESm09hre jet. The maxima of $(-$ Cup) and Cuиp obtained with the new velocity fields are presented in table 3. They clearly become lower as the intensity of the random disturbances increases.

\subsubsection{Case $M_{40} /$ shear-layer}

The normalized correlations between fluctuating pressure at the downstream observation point $M_{40}$ and the signals of fluctuating velocity $u^{\prime}$ and normal stresses $v^{\prime 2}$ and $w^{\prime 2}$ along the shear layer are represented in figure 11 . The correlations obtained with other turbulence quantities or for the LESm06hre jet are not shown because they are very small. The correlations presented are themselves weak. At high Reynolds number in figures 11(a), they seem negligible, which agrees well with the 
(i)
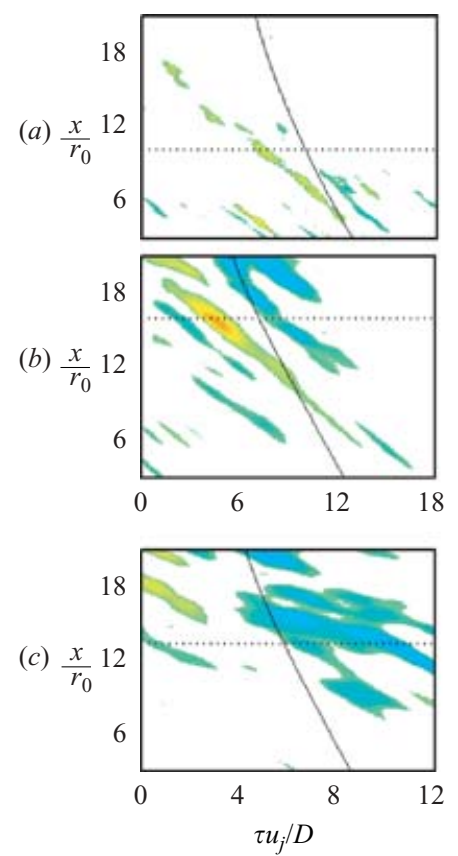

(ii)
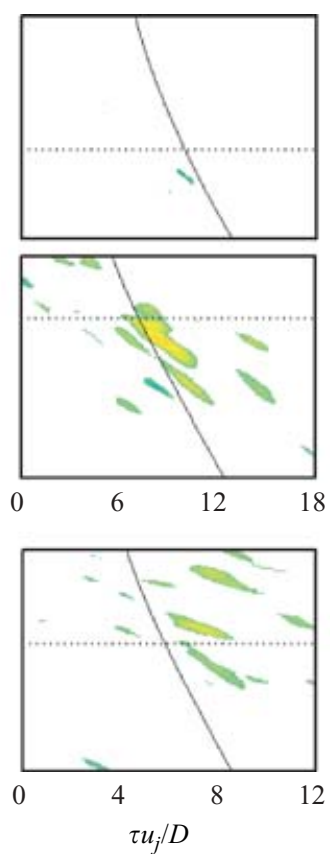

(iii)
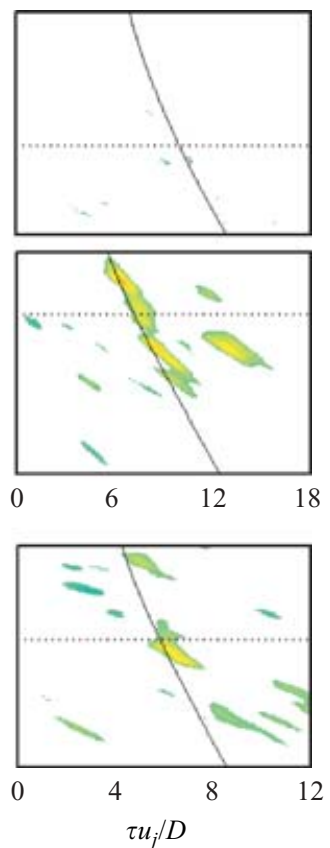

FIGURE 11. Case $M_{40} /$ shear-layer. Normalized correlations between pressure at $M_{40}$ and flow quantities along the shear layer: (i) Cup, (ii) Cvvp, (iii) Cwwp, for (a) LESm09hre, (b) LESm09re1700, (c) LESm06re1700 ( $X$-axis: time delay $\tau u_{j} / D, Y$-axis: axial location $x / r_{0}$ along the shear layer). The solid lines represent the propagation time $\tau_{\text {ray }}$ between the shear-layer points and the point $M_{40}$. See caption of figure 6 for colour scale and dotted lines.

measurements of Panda \& Seasholtz (2002) and Panda et al. (2005) displaying very low correlations between shear-layer turbulence and pressure at $30^{\circ}$ to the jet axis for a high-Reynolds-number jet at $M=0.95$. At $R e_{D}=1700$ in figures $11(b)$ and $11(c)$, the correlation levels are higher, especially for $C v v p$ and $C w w p$ calculated from $v^{\prime 2}$ and $w^{\prime 2}$. In these two cases, the peak correlations appear in the vicinity of the end of the potential core, at time delays in good agreement with the propagation times $\tau_{\text {ray }}$, as in the previous case $M_{40} /$ jet axis. The correlation values exhibited in the case $M_{40} /$ shear-layer are nevertheless significantly lower.

The latter observations are supported in figure 12 by the axial variations of the peaks of $C v v p$ and $C w w p$. At high Reynolds number, no significant peak can be distinguished from the background noise of about 0.03. At the low Reynolds number $R e_{D}=1700$, the correlation levels are higher in the region near the end of the jet core. The maximum correlations are about 0.06 for the jet at Mach 0.9 and 0.05 for the jet at Mach 0.6. These values are noticeably lower than the maximum correlations obtained between pressure at point $M_{40}$ and normal stresses along the jet centreline, which are about 0.14 for the LESm09re1700 jet and 0.10 for the LESm06re1700 jet (table 2).

\subsection{Correlations between pressure at $M_{90}$ and jet turbulence}

\subsubsection{Case $M_{90} /$ shear-layer}

The normalized cross-correlations between pressure at point $M_{90}$ at $90^{\circ}$ to the jet axis, relative to the end of the potential core, and signals of fluctuating velocity $u^{\prime}$, 

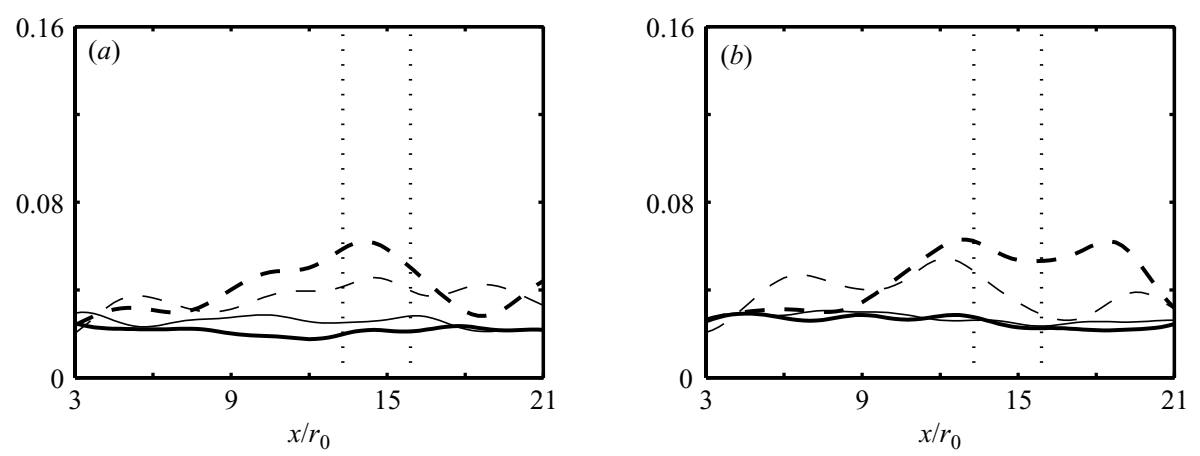

FIGURE 12. Case $M_{40} /$ shear-layer. Axial variation of peak correlation between pressure at $M_{40}$ and flow quantities along the shear layer. Peaks of: (a) $C v v p,(b) C w w p$, for LESm09hre; -- -, LESm09re1700; - , LESm06hre; ---, LESm06re1700. The dotted lines show the end of the potential core for the jets at $R e_{D}=1700$.

and normal stresses $u^{\prime 2}$ and $v^{\prime 2}$, along the shear layer, are presented in figure 13 for the two jets at Mach 0.9. The correlation levels are found to be very weak for the jet at high Reynolds number in figure $13(a)$, as well as for the jet at $R_{D}=1700$ in figure $13(b)$. Note that negligible correlations are also observed using any other turbulence quantity in the shear layer, and for the jets at Mach 0.6.

Exhibiting causality links between sideline pressure and shear-layer turbulence therefore appears difficult using broadband direct cross-correlations, although measurements, from Zaman (1986) in particular, suggested that a significant part of sideline noise is generated in the jet shear layer. The low correlation levels in the case $M_{90} /$ shear layer certainly arise from the jet noise component predominant in the sideline direction being due the randomly developing turbulence, see for instance in Tam et al. (1996) and Bogey \& Bailly (2006b), over an extended source region as pointed out by Seiner (1974).

\subsubsection{Case $M_{90} /$ axis}

Finally, the normalized correlations between the fluctuating pressure at the sideline point $M_{90}$ and the turbulence signals $u^{\prime}, u^{\prime 2}$ and $|\boldsymbol{\omega}|$ along the jet axis are presented in figure 14 for the two jets at Mach 0.9 and for LESm06re1700. For brevity, the correlations calculated from the normal stresses $v^{\prime 2}, w^{\prime 2}$ and from the turbulent kinetic energy $k$ are not provided. Note, however, that they show correlation fields similar to those obtained from $|\omega|$ in figures 14(a)(iii)-14(c)(iii). The correlation levels are negligible for the high-Reynolds-number jet in figure 14(a), but they are found to be significant for the jets at $R e_{D}=1700$ in figures $14(b)$ and $14(c)$. In the latter case, large zones with correlation levels higher than 0.04 are observed. As with the correlations involving the downstream observation point $M_{40}$, the maximum levels are observed in the vicinity of the end of the potential core. They also occur for time delays fairly close to the propagation times between the aerodynamic and acoustic observation points considered. Nevertheless, with respect to the case $M_{40} /$ axis in $\S 3.1 .1$, in which centreline turbulence is correlated with the pressure emitted at $40^{\circ}$ to the jet axis, the peaks are of opposite signs. Compare for instance the Cuup correlations obtained for the LESm09re1700 jet, reported in figure 14(b)(ii) in the present case and in figure $6(b)$ (ii) in the case $M_{40} /$ axis: negative and positive peaks are observed, respectively. 
(i)

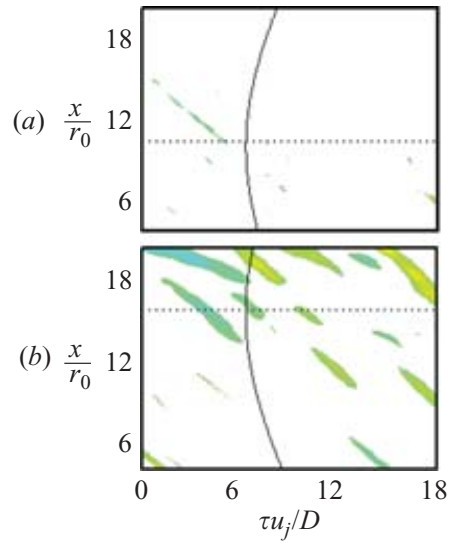

(ii)
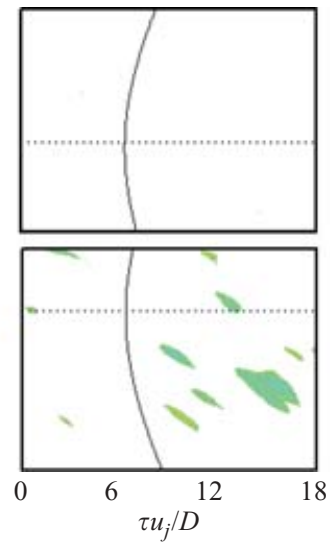

(iii)
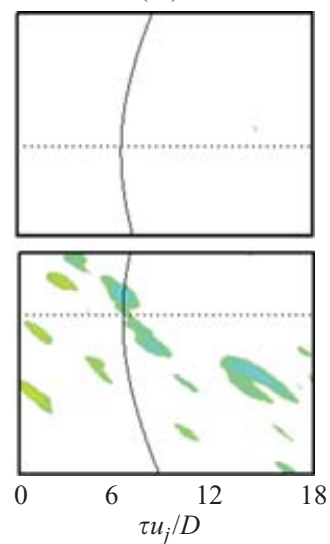

FIGURE 13. Case $M_{90}$ /shear-layer. Normalized correlations between pressure at $M_{90}$ and flow quantities along the shear layer: (i) Cup, (ii) Cuup, (iii) Cvvp, for (a) LESm09hre, (b) LESm09re1700 ( $X$-axis: time delay $\tau u_{j} / D, Y$-axis: axial location $x / r_{0}$ along the shear layer). The solid lines represent the propagation time $\tau_{\text {ray }}$ between the shear-layer points and point $M_{90}$. See caption of figure 6 for colour scale and dotted lines.

(i)
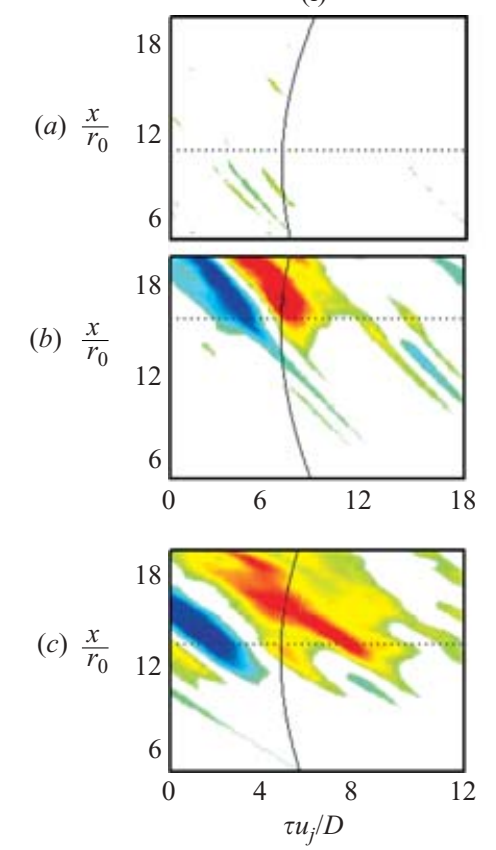

(ii)
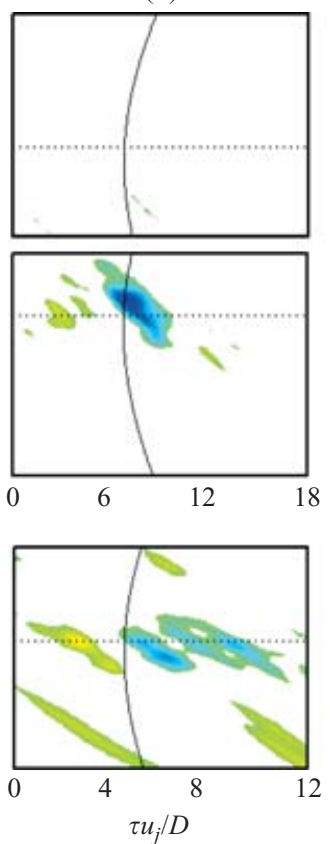

(iii)
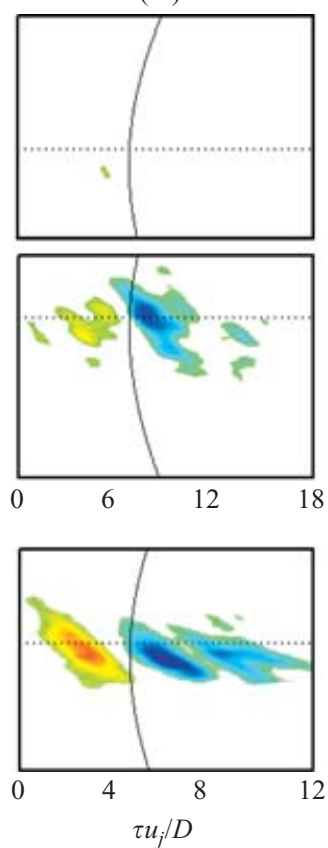

Figure 14. Case $M_{90}$ /axis. Normalized correlations between pressure at $M_{90}$ and flow quantities along the jet axis: (i) Cup, (ii) Cuup, (iii) $C|\omega| p$, for (a) LESm09hre, (b) LESm09re1700, (c) LESm06re1700 ( $X$-axis: time delay $\tau u_{j} / D, Y$-axis: axial location $x / r_{0}$ along the jet axis). The solid lines represent the propagation time $\tau_{\text {ray }}$ between the centreline points and point $M_{90}$. See caption of figure 6 for colour scale and dotted lines.

The correlations between the fluctuating pressure at point $M_{90}$ and the signals of velocity $u^{\prime}$ at $x=x_{c}+r_{0}$, and of $u^{\prime 2}$ and $|\omega|$ at $x=x_{c}-r_{0}$ on the jet axis are now presented in figure 15 for the two jets at $\operatorname{Re}_{D}=1700$. The correlation functions $C u p$ 
(i)

(a)

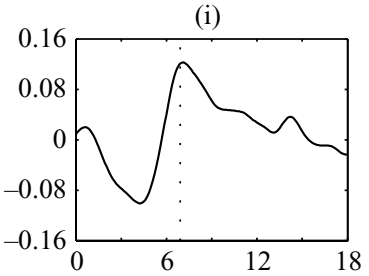

(b)

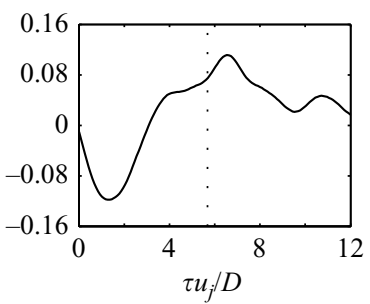

(ii)
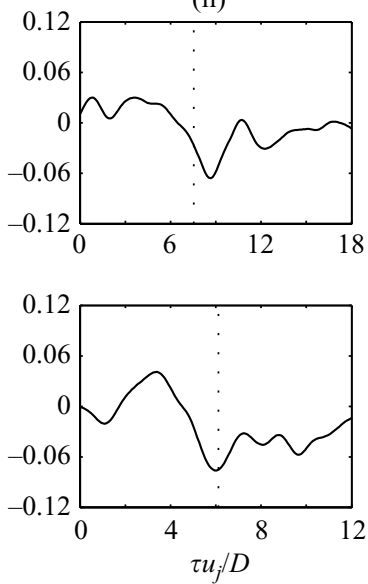

(iii)
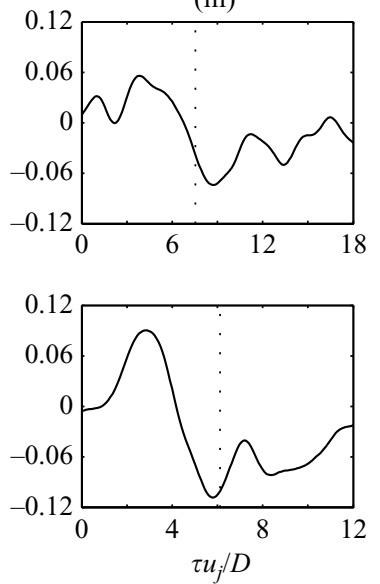

FIgURE 15. Case $M_{90} /$ axis. Normalized correlations between pressure at $M_{90}$ and flow quantities on the jet axis: (i) $C u p$ for a centreline point at $x=x_{c}+r_{0}$, (ii) $C u u p$ and (iii) $C|\omega| p$ for a point at $x=x_{c}-r_{0}$, for $(a)$ LESm09re1700, (b) LESm06re1700 $\left(X\right.$-axis: time delay $\left.\tau u_{j} / D\right)$. The dotted lines show the propagation time $\tau_{\text {ray }}$ between the centreline point and point $M_{90}$.

show a positive peak at about the propagation time delay, preceded by a negative peak with a similar level. The correlation functions Сиup and $C|\omega| p$ display the opposite case: the peak nearly at the propagation time is negative, and the preceding peak is positive. As for the peak levels, they are about $0.08-0.12$, and appear lower than those obtained in the case $M_{40}$ /axis which are 0.23 for ( $-C u p$ ) and 0.17 for $C|\omega| p$, for the LESm09re1700 jet. In addition, no appreciable effect of the Mach number can be convincingly found between the present results at Mach 0.9 and Mach 0.6.

\subsection{Properties of centreline turbulence}

In this section, the properties of the centreline turbulence, for which the maximum correlations are obtained with the radiated pressure, are investigated.

\subsubsection{Spectral properties}

The spectral content of the centreline turbulence is first studied. The spectra of the fluctuating radial velocity $v^{\prime}$ obtained on the jet axis at $x=x_{c}$ at the end of the potential core are presented in figures $16(a)$ and $16(b)$ for the jets at Mach 0.9 and Mach 0.6, respectively. At both Mach numbers, the spectra are dominated by frequency peaks at the low Strouhal numbers of $S t \simeq 0.3$ at the high Reynolds numbers $R e_{D} \geqslant 10^{5}$, and of $S t \simeq 0.2$ at $R e_{D}=1700$. The peaks are, however, more marked for the low-Reynolds-number jets. The peak frequencies in the present velocity spectra at the end of the jet core are in good agreement with the peak Strouhal numbers in the sound pressure spectra obtained at about $30^{\circ}$ to the jet axis (Bogey \& Bailly 2006b). They further agree with the peaks observed in the pressure spectra at $40^{\circ}$ to the jet axis for the $M=0.9$ jets (figure $3 a$ ).

The magnitudes and Strouhal numbers associated with the peaks in the spectra of centreline velocity $v^{\prime}$ are shown in figure 17 for $x_{c}-4 r_{0} \leqslant x \leqslant x_{c}+4 r_{0}$. The increase of the peak magnitudes in figure 17(a) shows the rapid growth of the turbulence intensity at the end of the potential core. For the different jets, the peak Strouhal number is found (figure 17b) not to vary much in the considered flow region, extending from two diameters upstream of the end of the potential core up to two diameters downstream. 

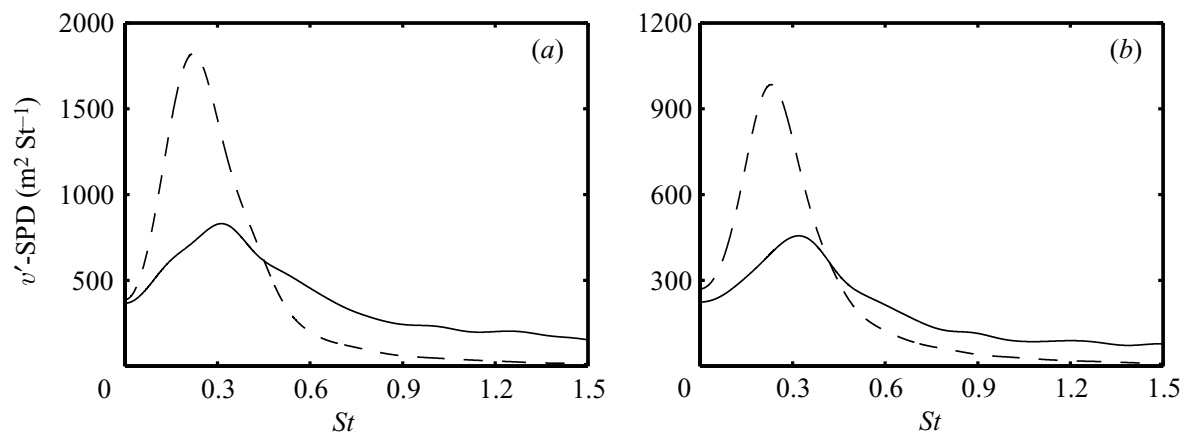

FIGURE 16. Spectral power densities of $v^{\prime}$-velocity as a function of Strouhal number $S t=$ $f D / u_{j}$, at $x=x_{c}$ on the jet axis: (a) Mach 0.9 jets, (b) Mach 0.6 jets; at $\longrightarrow$, high Reynolds number; ---, $R e_{D}=1700$.
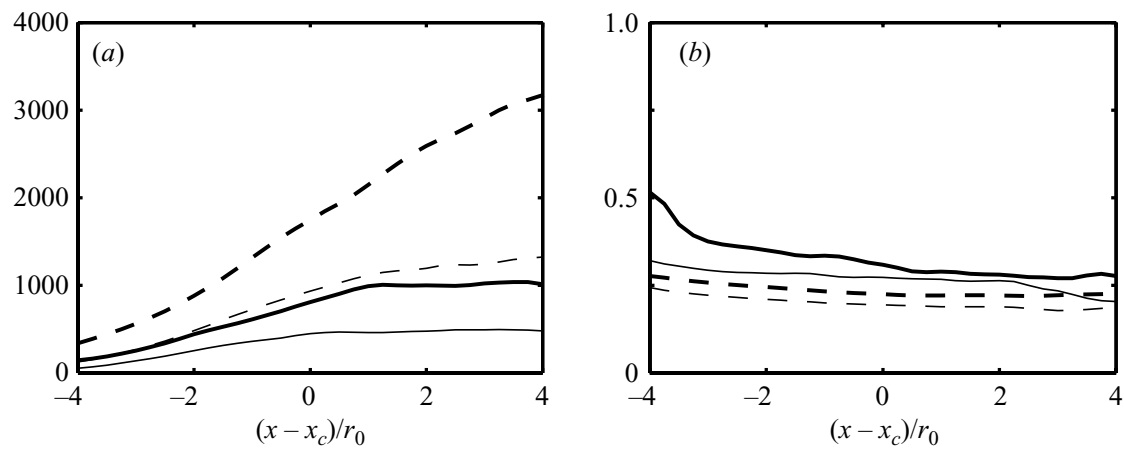

Figure 17. (a) Level (in $\mathrm{m}^{2}$ per $S t$ ) and $(b)$ Strouhal number of the peak in $v^{\prime}$-velocity spectra on the jet axis, as a function of $\left(x-x_{c}\right) / r_{0}$, the axial position with respect to the end of the potential core, for: - - LESm09hre; ---, LESm09re1700; —-, LESm06hre; ---, LESm06re1700.

This observation suggests the occurrence, at the end of the potential core on the jet centreline, of a turbulence phenomenon that is persistent over a large axial distance.

\subsubsection{Intermittency}

The features of the flow phenomena at the end of the potential core are now investigated through intermittency, as for instance in Sabot \& Comte-Bellot (1976). The intermittency of momentum and heat transport in turbulent round jets was studied, for example, by Chevray \& Tutu (1978), and that of coherent structures in the near field of a high-Reynolds-number turbulent jet flow was examined by Camussi \& Guj (1999). The intermittency of the noise emission in subsonic jets was also shown by Juvé et al. (1980) using a conditional sampling procedure in the calculation of correlations between far-field acoustic pressure at $30^{\circ}$ to the jet direction and velocity fluctuations in a $M=0.4$ jet.

In the present work, the intermittency of the centreline turbulence is analysed by means of the function $I(t)$ defined arbitrarily from the vorticity norm $|\omega|$ by

$$
I(t)= \begin{cases}1 & \text { if }|\omega| \text { is lower than }\langle|\boldsymbol{\omega}|\rangle / 2, \\ 0 & \text { otherwise, }\end{cases}
$$




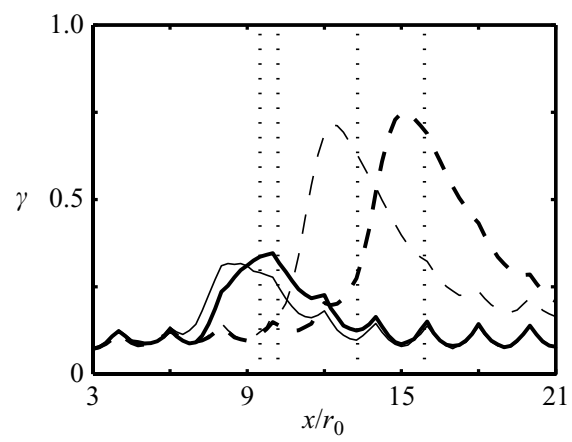

FIGURE 18. Intermittency factor $\gamma$ along the jet axis, calculated from the vorticity norm $|\omega|$ : —, LESm09hre; ---, LESm09re1700; —, LESm06hre; ---, LESm06re1700. The dotted lines show the end of the potential core for the different jets.

which allows us to determine the intermittency factor $\gamma=\langle I(t)\rangle$. This factor is based on the vorticity norm, but normal stresses or turbulent kinetic energy could be used in the same way. One expects that $\gamma \simeq 0$ when the vorticity signal is laminar or turbulent, but that $\gamma \simeq 1$ when the signal is intermittent.

The factor $\gamma$, calculated along the centreline of the present jets from the vorticity norm as described above, is represented in figure 18. For the four jets, it is about 0.1 in the first part of the potential core, and displays a noticeable increase near the end of the core, up to about 0.75 for the LESm09re1700 jet, with a maximum located just upstream of $x=x_{c}$. Farther downstream, the factor $\gamma$ decreases progressively to recover finally values close to 0.1 . The vorticity signal is therefore significantly intermittent in the vicinity of the end of the jet core. The intermittency level is found to be slightly higher at Mach 0.9 than at Mach 0.6. It also rises appreciably as the Reynolds number is reduced down to $R e_{D}=1700$. Moreover, there is a good similarity between the variations of the intermittency of centreline vorticity norm $|\omega|$ in figure 18, and those of the peak of the correlations $C|\boldsymbol{\omega}| p$ between pressure at point $M_{40}$ and centreline vorticity in figure 10 . The higher the intermittency of the vorticity signal at the end of the potential core, the higher the maximum correlation that is observed.

The intermittency of the centreline turbulence is illustrated in figure 19(a) displaying the time evolution of vorticity norm $|\omega|$ at $x=x_{c}$ on the jet axis for the two Mach number 0.9 jets. In both jets, turbulence appears to be intermittently, and periodically, present on the jet axis at the end of the potential core. The associated non-dimensional periods are visibly around 3 for the jet at $R e_{D} \geqslant 10^{5}$, and 5 for the jet at $R e_{D}=1700$, which is in good agreement with the peak Strouhal numbers $S t \simeq 0.3$ and $S t \simeq 0.2$ obtained in the spectra of velocity $v^{\prime}$ in figure 16(a). The phenomenon of intrusion of vortical structures into the jet core is thus observed at both high and low Reynolds numbers, regardless of the absence or presence of small turbulent scales. It was previously described from results of a first simulation in Bogey et al. (2003), and was connected to the sound waves radiated in the downstream direction. The present results strongly support this view. The deterministic source responsible for the downstream jet noise component, discussed in Bogey \& Bailly (2006b), is therefore likely to be this mechanism at the end of the potential core.

To show how the intermittent signals of turbulence can provide significant correlations with the pressure radiated downstream, the time evolution of vorticity $|\omega|$ at 

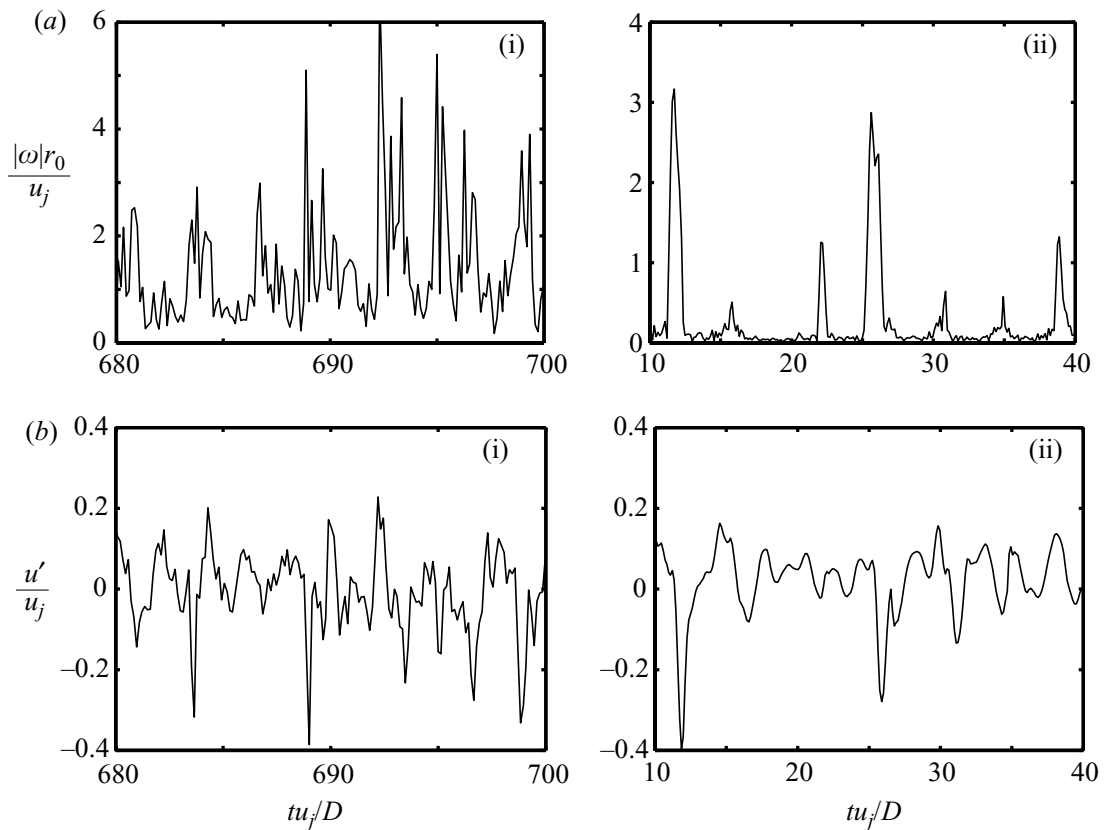

FIGURE 19. Time evolution of the vorticity norm $|\omega| \times r_{0} / u_{j}(a)$ and of the fluctuating axial velocity $u^{\prime} / u_{j}(b)$, at $x=x_{c}$ on the jet axis. (i) for LESm09hre, (ii) for LESm09re1700.

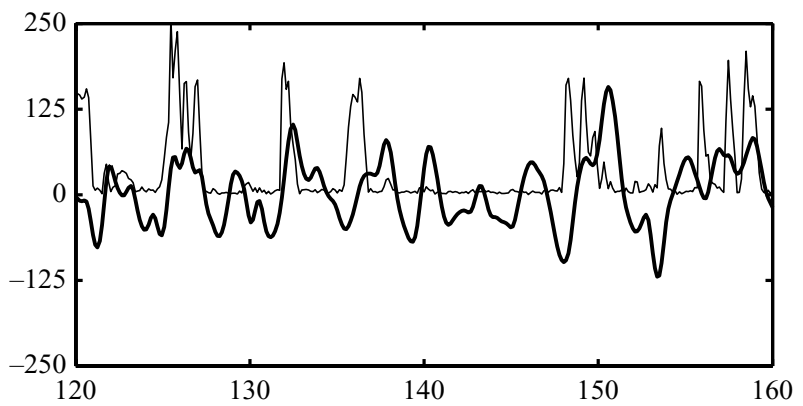

FIGURE 20. Time evolution, for LESm09re1700: - vorticity $|\boldsymbol{\omega}| \times r_{0} / u_{j} \times 70$ at $x=x_{c}$ on the jet axis, at time $t u_{j} / D$; - fluctuating pressure $p^{\prime}$ (in Pa) at point $M_{40}$, at the retarded time $\left(t-\tau_{\text {ray }}\right) u_{j} / D\left(\tau_{\text {ray }}\right.$ is the propagation time between the centreline point and $\left.M_{40}\right)$.

$x=x_{c}$ on the jet axis is represented in figure 20 for the LESm09re1700 jet. The signal of fluctuating pressure $p^{\prime}$ at point $M_{40}$ is also given at the retarded time taking account of the propagation time delay $\tau_{\text {ray }}$ between the source and observation points. The bursts of vorticity appear to coincide with positive pressure fronts, which yield the positive correlation peaks obtained in figures 7(b)(iii) and $9(b)$ (iii). Identical observations could be made with the signals of normal stresses and turbulence kinetic energy, which all are positive quantities, and exhibit similar cross-correlations with the radiated pressure.

For the fluctuating axial velocity, the case is different: the intrusion of vortical structures into the jet core results in a velocity deficit. Negative peaks of $u^{\prime}$ then occur intermittently. They are clearly observed in figure $19(b)$ where the signals of velocity $u^{\prime}$ 
are represented for the two jets at Mach 0.9 at the same locations and times as the vorticity norm in figure $19(a)$. In both jets, the negative peaks of $u^{\prime}$ are strong with amplitudes that can reach $40 \%$ of the jet inflow velocity, and they seem more marked than those attributed only to random turbulent motions. These peaks of $u^{\prime}$, being obtained at the same time as the peaks of vorticity norm, will correlate with positive fronts of the pressure signal at point $M_{40}$ provided that the same appropriate retarded time is used. This will yield the negative correlations calculated between centreline velocity and downstream pressure, which are exhibited for instance in figures $6(b)(i)$ and 9(b)(i) for the LESm09re1700 jet. These results agree with the experimental observations of Juvé et al. (1980) who noticed that, at points located near the end of the potential core, the periods of noise generation appear to coincide frequently with a marked deceleration of the flow. Note, finally, that no remarkable deviations due to the intermittency at the end of the potential core are observed in the signals of the fluctuating velocities $v^{\prime}$ and $w^{\prime}$. Fluctuations of $v^{\prime}$ and $w^{\prime}$ therefore seem to be associated only with the random motions of vortical structures, which leads to weak cross-correlations with the radiated pressure.

As for the correlations observed for the jets at the low Reynolds number $\operatorname{Re}_{D}=1700$ between centreline turbulence and sideline pressure at point $M_{90}$, their different shapes with respect to the case $M_{40}$ /axis may be linked to the features of the pressure signals at $90^{\circ}$ to the jet axis. These cross-correlations may further indicate that the intermittent jet-noise source does not contribute in a negligible manner to the radiated sideline noise at the low Reynolds number of $R e_{D}=1700$.

\subsubsection{Convection velocity}

In figures 6 and 7, the peak correlations between centreline turbulence and fluctuating pressure at point $M_{40}$ are located at the end of the potential core, for a time delay corresponding to the propagation time $\tau_{\text {ray }}$ along the ray path between the source and the observation points. However, the correlation fields do not seem to be aligned with the propagation times $\tau_{\text {ray }}$. This may indicate that the correlations obtained before and after the end of the jet core are associated with a flow phenomenon other than noise generation alone.

To study this issue, the convection velocity $u_{\text {conv }}$ along the jet axis is calculated from the correlation functions between signals of velocity $u^{\prime}$ at two points separated by the distance $r_{0}$. It is represented in figure 21 for the different jets. In the four cases, the velocity $u_{\text {conv }}$ is about $(2 / 3) \times u_{j}$ in the first part of the potential core, which is classically indicative of the convection of turbulent structures within the shear layer of round jets. The convection velocity, however, increases with the axial distance, reaches a peak slightly before the end of the potential core, and then decreases progressively. The maximum of convection velocity appears poorly affected by the Mach number, but it depends appreciably on the Reynolds number. For the Mach 0.9 jets, the peak velocity is for example $u_{\text {conv }}=0.97 u_{j}$ at $R e_{D} \geqslant 10^{5}$, which is very close to the jet inflow velocity, but $u_{\text {conv }}=0.81 u_{j}$ at $R e_{D}=1700$. In all the cases, the centreline turbulence is convected around the end of the potential core at a velocity significantly higher than the classical convection velocity inside the shear layer. Vortical structures intruding into the jet core are therefore strongly accelerated in the axial direction. According to the theory of vortex sound, formulated in particular by Powell (1964), we can expect this acceleration to be an effective noise generation mechanism in the jets.

The correlation fields obtained in the case $M_{40} /$ axis are now examined by constructing a new time delay between the centreline points and the observation point $M_{40}$. This time delay is equal to $\tau_{\text {ray }}$ at the end of the potential core, where the 


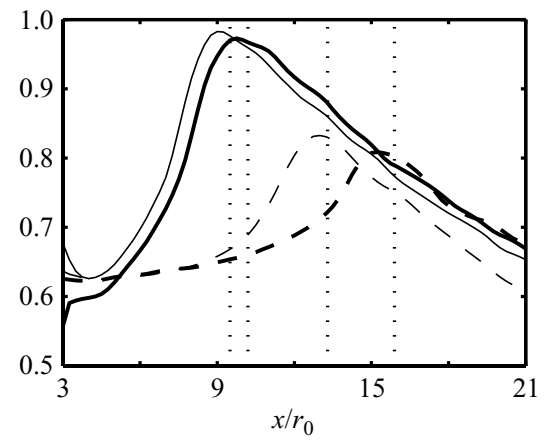

FIGURE 21. Convection velocity $u_{\text {conv }} / u_{j}$ along the jet axis:

, LESm09hre; --LESm09re1700; , LESm06hre; of the potential core for the different jets.

(i)
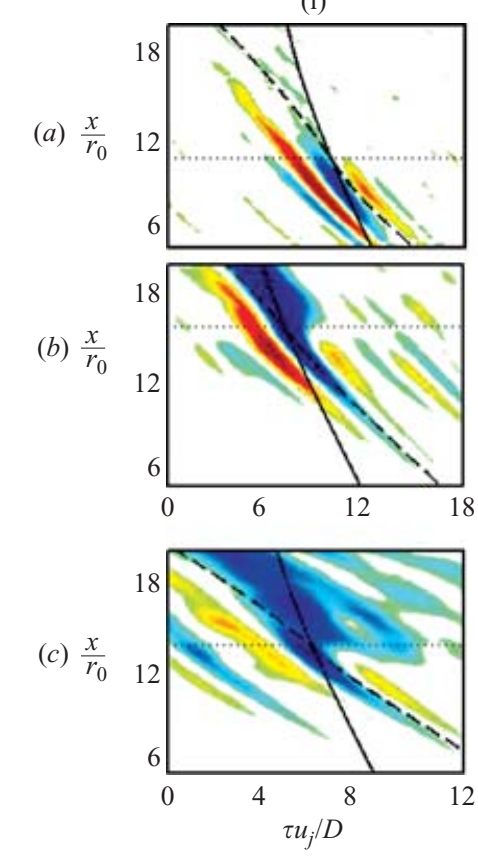

(ii)
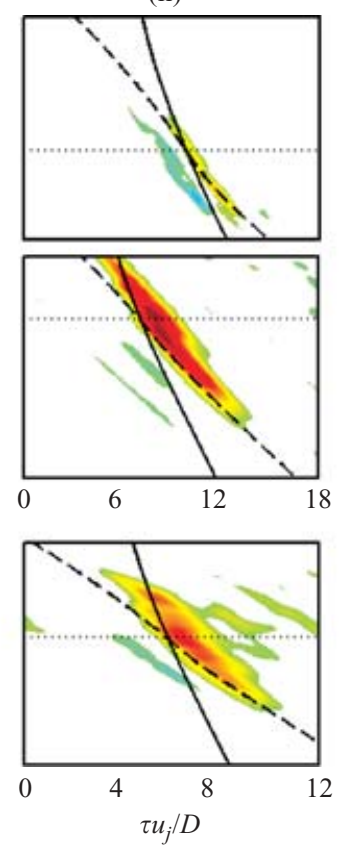

(iii)
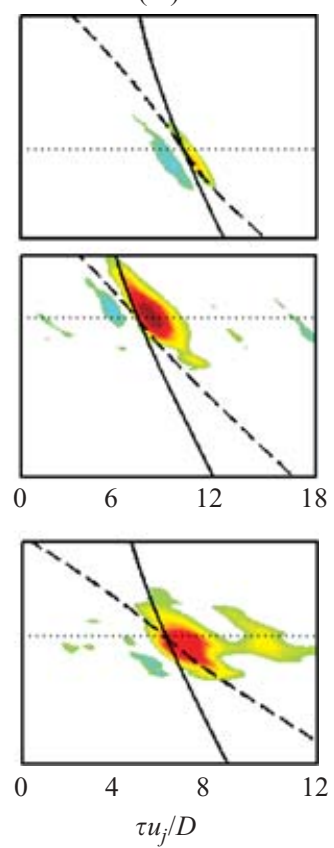

Figure 22. Case $M_{40}$ /axis. Normalized correlations: (i) Cup, (ii) $C v v p$, (iii) $C|\omega| p$, for (a) LESm09hre, (b) LESm09re1700, (c) LESm06re1700. See caption of figure 6 for details. The solid lines represent the propagation time $\tau_{\text {ray }}$ along the ray paths. The dashed lines show the time delay based on the convection velocity, egal to $\tau_{\text {ray }}$ at the end of the potential core.

peak correlation is observed at the propagation time delay along the ray path, and takes into account the convection velocity on the jet centreline, yielding:

$$
\tau(x)=\tau_{\text {ray }}\left(x_{c}\right)+\int_{x}^{x_{c}} \frac{\mathrm{d} x}{u_{\text {conv }}(x)} .
$$

In figure 22, the normalized correlations between fluctuating pressure at $M_{40}$ and the turbulence signals $u^{\prime}, v^{\prime 2}$ and $|\omega|$ along the jet axis are represented for the two jets at Mach 0.9 and for the LESm06re1700 jet. In all the cases, the trajectories of 

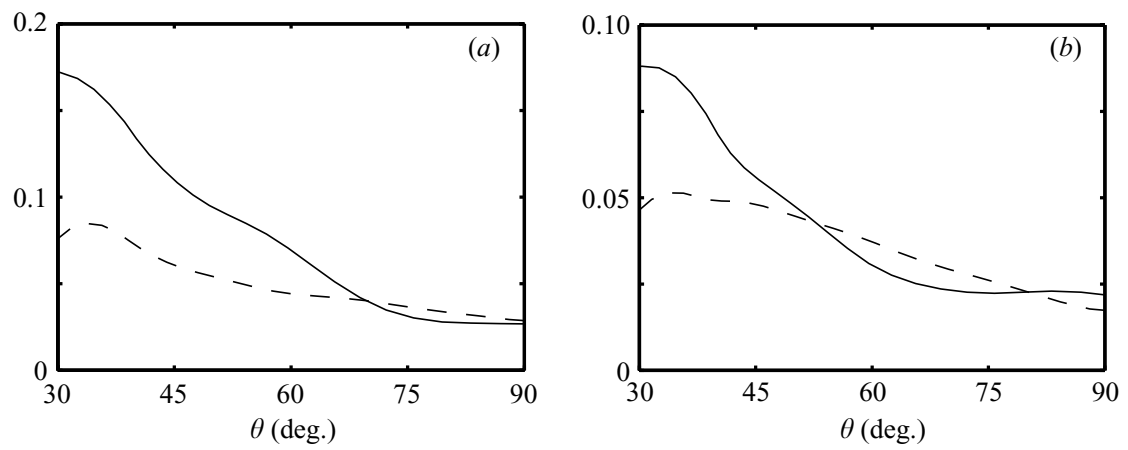

FIGURE 23. Angular variation of peak correlation between radiated pressure and flow quantities at $x=x_{c}$ on the jet axis ( $\theta$ is the angle with respect to the jet direction). Peaks of $(a) C u p$, (b) $C|\boldsymbol{\omega}| p$, for the high-Reynolds-number jets: — , LESm09hre; ---, LESm06hre.

the correlations are found to follow the time delay based on the convection velocity. The agreement is shown in figure $22(b)$ by the correlations Cup and Cvvp calculated for the LESm09re1700 jet. Thus, strong correlations between the flow and acoustic fields do not necessarily indicate significant noise emission. In the present case, the correlation fields suggest that one sound source is present, and convected, along the jet axis, and that the noise radiated by this source originates mostly from the region close to the end of the potential core. Noise generation therefore occurs mainly in the zone where the intermittency and acceleration of vorticity in the jet core are the highest.

\subsection{Correlations between pressure and turbulence at the end of the jet core}

The variations with the emission angle of the correlations between sound pressure and centreline turbulence at the end of the potential core are finally investigated. With this aim in view, normalized correlations are calculated between turbulence signals at $x=x_{c}$ on the jet axis, and fluctuating pressure at the different acoustic observation points presented in figure 1 . The peaks of the cross-correlations Cup and $C|\omega| p$ computed from velocity $u^{\prime}$ and vorticity norm $|\omega|$ are plotted in figures $23(a)$ and $23(b)$, as functions of the emission angle $\theta$ relative to the end of the jet core, for the high-Reynolds-number jets LESm09hre and LESm06hre at $M=0.9$ and $M=0.6$. In both jets, the correlation levels are significant for the pressure radiated in the downstream direction, but they are observed to decrease dramatically with the emission angle. They are particularly low for $\theta \geqslant 75^{\circ}$. This behaviour is in agreement with the experimental data provided for jets at high Reynolds number by Hurdle et al. (1974) and Panda et al. (2005).

The angular dependence of the present correlations between pressure and turbulence at the end of the jet core brings us some information about the contribution of the intermittent sound source to the radiated noise for the high-Reynolds-number jets considered. This contribution appears to be very important at small radiation angles, but small in the sideline direction, which is in agreement with the source directivity suggested by other works, especially by Tam et al. (1996) and Bogey \& Bailly (2006b).

\section{Conclusion}

In the present paper, noise generation is investigated using causality methods in subsonic isothermal round jets computed by large eddy simulation, at Mach numbers 
$M=0.6$ and $M=0.9$, at the low and high Reynolds numbers of $R e_{D}=1700$ and $R e_{D} \geqslant 10^{5}$. Normalized correlations between the broadband signals of jet turbulence and of radiated pressure, provided directly by the simulations, are presented. The turbulence quantities considered are the fluctuating velocities, the normal stresses, the turbulent kinetic energy and the vorticity norm. Correlations are calculated using the pressure obtained at the radiation angles, relative to an origin at the end of the potential core, of $40^{\circ}$ and $90^{\circ}$ from the jet direction, and turbulence along the jet axis and along the shear layer.

For the pressure radiated at $40^{\circ}$ to the jet axis, significant levels of correlation are obtained with the centreline turbulence in all the simulated jets. Peak correlations are observed at the end of the potential core, for time delays corresponding fairly well to the times of propagation along the ray paths between the source and the emission points. They are between 0.06 and 0.15 for the jets at high Reynolds numbers $R e_{D} \geqslant 10^{5}$. They are higher for the jets at the low Reynolds number of $R e_{D}=1700$, and decrease as the Mach number is reduced. The correlation fields are also shown to be connected to the convection velocity along the jet centreline. The correlations between pressure at $40^{\circ}$ to the jet axis and turbulence along the shear layer are considerably smaller. They reach a maximum of only 0.06 for the jets at $R e_{D}=1700$, and they are very small for the high-Reynolds-number jets.

For the sideline pressure at $90^{\circ}$ to the jet axis, the levels of correlations with the shear-layer turbulence are found to be insignificant in all the computed jets. The cross-correlations with the centreline turbulence are also weak, especially for the high-Reynolds-number jets. They are, however, appreciable for the jets at $\operatorname{Re}_{D}=1700$. In that case, maximum correlation levels of about 0.10 are obtained at the end of the jet core.

The present results support the presence of a noise generation mechanism near the end of the potential core, on the jet centreline. The correlations moreover suggest that the contribution of this sound source to the radiated noise is important in the downstream direction, but very small in the sideline direction, except at very low Reynolds numbers. Considering this feature, the noise source in question is probably the source responsible for the downstream jet-noise component exhibited in experimental and numerical works. The signals of turbulence at the end of the jet core show that this source is characterized by a dominant low-Strouhal-number frequency over a large axial distance and by a high level of intermittency. The periodic and intermittent intrusion of vortical structures into the jet core, and consequently their sudden acceleration, can therefore be expected to be the turbulent phenomenon involved in this noise generation mechanism.

This work was partly supported by the ANR (the French national research agency) Program NT05-4_42264 'Identification des mécanismes générateurs de bruit dans les écoulements libres'. The authors gratefully acknowledge the Institut du Développement et des Ressources en Informatique Scientifique (IDRIS-CNRS) for providing computing time and for its technical assistance.

\section{REFERENCES}

Arakeri, V. H., Krothapalli, A., Siddavaram, V., Alkislar, M. B. \& Lourenco, L. 2003 On the use of microjets to suppress turbulence in a Mach 0.9 axisymmetric jet. J. Fluid Mech. 490, $75-98$.

Arndt, R. E. A., Long, D. F. \& Glauser, M. N. 1997 The proper orthogonal decomposition of pressure fluctuations surrounding a turbulent jet. J. Fluid Mech. 340, 1-33. 
Bogey, C., Barré, S., Fleury, V., Bailly, C. \& Juvé, D. 2007 Experimental study of the spectral properties of near-field and far-field jet noise. Intl J. Aeroacoust. 6(2) (in press).

Bogey, C. \& Bailly, C. 2004 A family of low dispersive and low dissipative explicit schemes for flow and noise computations. J. Comput. Phys. 194, 194-214.

Bogey, C. \& Bailly, C. 2005 a Effects of inflow conditions and forcing on a Mach 0.9 jet and its radiated noise. AIAA J. 43, 1000-1007.

Bogey, C. \& Bailly, C. $2005 b$ Decrease of the effective Reynolds number with eddy-viscosity subgrid-scale modeling. AIAA J. 43, 437-439.

Bogey, C. \& Bailly, C. $2006 a$ Computation of a high Reynolds number jet and its radiated noise using LES based on explicit filtering. Computers Fluids 35, 1344-1358.

Bogey, C. \& Bailly, C. $2006 b$ Investigation of downstream and sideline subsonic jet noise using large eddy simulation. Theor. Comput. Fluid Dyn. 20, 23-40.

Bogey, C. \& Bailly, C. 2006 c Large eddy simulations of round free jets using explicit filtering with/without dynamic Smagorinsky model. Intl J. Heat Fluid Flow 27, 603-610.

Bogey, C. \& Bailly, C. $2006 d$ Large eddy simulations of transitional round jets: influence of the Reynolds number on flow development and energy dissipation. Phys. Fluids 18(6), 065101.

Bogey, C., Bailly, C. \& JuvÉ, D. 2003 Noise investigation of a high subsonic, moderate Reynolds number jet using a compressible LES. Theor. Comput. Fluid Dyn. 16, 273-297.

CAmussi, R. \& Guj, G. 1999 Experimental analysis of intermittent coherent structures in the near field of a high Re turbulent jet flow. Phys. Fluids 11(2), 423-431.

CANDEL, S. M. 1977 Numerical solution of conservation equations arising in linear wave theory: application to aeroacoustics. J. Fluid Mech. 83, 465-493.

Chevray, R. \& Tutu, N. K. 1978 Intermittency and preferential transport of heat in a round jet. J. Fluid Mech. 88, 133-160.

Chu, W. T. \& KaPlan, R. E. 1976 Use of a spherical concave reflector for jet-noise-source distribution diagnosis. J. Acoust. Soc. Am. 59, 1268-1277.

Coiffet, F., Jordan, P., Delville, J., Gervais, Y. \& Ricaud, F. 2006 Coherent structures in subsonic jets: a quasi-irrotational source mechanism? Intl J. Aeroacoust. 5, 67-89.

Crighton, D. G. 1981 Acoustics as a branch of fluid mechanics. J. Fluid Mech. 106, 261-298.

Dahan, C., Elias, G., Maulard, J. \& Perulli, M. 1978 Coherent structures in the mixing zone of a subsonic hot free jet. J. Sound Vib. 59, 313-333.

FREUND, J. B. 2001 Noise sources in a low-Reynolds-number turbulent jet at Mach 0.9. J. Fluid Mech. 438, 277-305.

Goldstern, M. E. 1984 Aeroacoustics of turbulent shear flows. Annu. Rev. Fluid Mech. 16, 263-285.

Hileman, J. \& Samimy, M. 2001 Turbulence structures and the acoustic far field of a Mach 1.3 jet. AIAA J. 39, 1716-1727.

Hileman, J. I., Thurow, B. S., Caraballo, E. J. \& Samimy, M. 2005 Large-scale structure evolution and sound emission in high speed jets: real-time vizualization with simulataneous acoustic measurements. J. Fluid Mech. 544, 277-307.

Hurdle, P. M., Meecham, W. C. \& Hodder, B. K. 1974 Investigation of the aerodynamic noise generating region of a jet engine by means of the simple source fluid dilatation model. J. Acoust. Soc. Am. 56, 1708-1721.

Juvé, D., Sunyach, M. \& Comte-Bellot, G. 1980 Intermittency of the noise emission in subsonic cold jets. J. Sound Vib. 71, 319-332.

LaU, J. C., Morris, P. J. \& Fisher, M. J. 1979 Measurements in subsonic and supersonic free jets using a laser velocimeter. J. Fluid Mech. 93, 1-27.

Lee, H. K. \& Ribner, H. S. 1972 Direct correlation of noise and flow of a jet. J. Acoust. Soc. Am. 52, 1280-1290.

Long, D. F. \& ARndt, R. E. A. 1984 Jet noise at low Reynolds number. AIAA J. 22, 187-193.

Lush, P. A. 1971 Measurements of subsonic jet noise and comparison with theory. J. Fluid Mech. 46, 477-500.

Mollo-Christensen, E., Kolpin, M. A. \& Martucelli, J. R. 1964 Experiments on jet flows and jet noise far-field spectra and directivity patterns. J. Fluid Mech. 18, 285-301.

Panda, J. \& Seasholtz, R. G. 2002 Experimental investigation of density fluctuations in high-speed jets and correlation with generated noise. J. Fluid Mech. 450, 97-130.

Panda, J., Seasholtz, R. G. \& Elam, K. A. 2005 Investigation of noise sources in high-speed jets via correlation measurements. J. Fluid Mech. 537, 349-385. 
Powell, A. 1964 Theory of vortex sound. J. Acoust. Soc. Am. 36, 177-195.

Richardz, W. G. 1980 Direct correlation of noise and flow of a jet using laser Doppler. AIAA J. 18, 759-765.

Rizzetta, D. P., Visbal, M. R. \& Blaisdell, G. A. 2003 A time-implicit high-order compact differencing and filtering scheme for large-eddy simulation. Intl J. Numer. Meth. Fluids 42, 665-693.

Sabot, J. \& Comte-Bellot, G. 1976 Intermittency of coherent structures in the core region of fully developed turbulent pipe flow. J. Fluid Mech. 74, 767-796.

SchafFAR, M. 1979 Direct measurements of the correlation between axial in-jet velocity fluctuations and far field noise near the axis of a cold jet. J. Sound Vib. 64, 73-83.

SEINER, J. M. 1974 The distribution of jet source strength intensity by means of direct correlation technique. PhD thesis, Pennsylvania State University.

Siddon, T. E. \& RACKL, R. 1971 Cross-correlation analysis of flow noise with fluid dilatation as source fluctuation. 82nd Meeting of the Acoustical Society of America, Denver, Oct. 1971.

Stromberg, J. L., Mclaughlin, D. K. \& Troutt, T. R. 1980 Flow field and acoustic properties of a Mach number 0.9 jet at a low Reynolds number. J. Sound. Vib. 72, 159-176.

TAM, C. K. W. 1998 Jet noise: since 1952. Theor. Comput. Fluid Dyn. 10, 393-405.

TAM, C. K. W., Golebiowski, M. \& Seiner, J. M. 1996 On the two components of turbulent mixing noise from supersonic jets. AIAA Paper 96-1716.

Ukeiley, L. S. \& Ponton, M. K. 2004 On the near field pressure of a transonic axisymmetric jet. Intl J. Aeroacoust. 3, 43-66.

Viswanathan, K. 2002 Analysis of the two similarity components of turbulent mixing noise. AIAA J. 40, 1735-1744.

Zaman, K. B. M. Q. 1985 Far-field noise of a subsonic jet under controlled excitation. J. Fluid Mech. 152, 83-111.

Zaman, K. B. M. Q. 1986 Flow field and near and far sound field of a subsonic jet. J. Sound Vib. 106, $1-16$.

Zaman, K. B. M. Q. \& Yu, J. C. 1985 Power spectral density of subsonic jet noise. J. Sound Vib. 98, $519-537$. 